\title{
Amorphization and Nanocrystallization of Silicon under Shock Compression
}

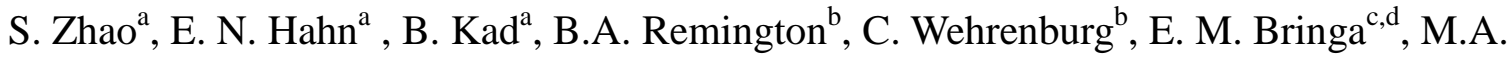 \\ Meyers $^{\mathrm{a}^{*}}$ \\ ${ }^{a}$ University of California, San Diego, La Jolla, CA 92093, USA \\ ${ }^{\mathrm{b}}$ Lawrence Livermore National Laboratory, Livermore, CA 94550, USA

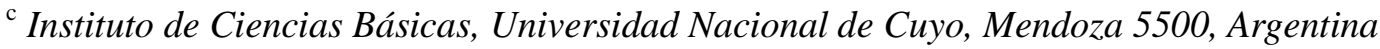 \\ ${ }^{d}$ CONICET, Mendoza 5500, Argentina
}

\begin{abstract}
High-power, short-duration, laser-driven, shock compression and recovery experiments on [001] silicon unveiled remarkable structural changes above a pressure threshold. Two distinct amorphous regions were identified: (a) a bulk amorphous layer close to the surface and (b) amorphous bands initially aligned with $\{111\}$ slip planes. Further increase of the laser energy leads to the re-crystallization of amorphous silicon into nanocrystals with high concentration of nano-twins. This amorphization is produced by the combined effect of high magnitude hydrostatic and shear stresses under dynamic shock compression. Shock-induced defects play a very important role in the onset of amorphization. Calculations of the free energy changes with pressure and shear, using the Patel-Cohen methodology, are in agreement with the experimental results. Molecular dynamics simulation corroborates the amorphization, showing that it is initiated by the nucleation and propagation of partial dislocations. The nucleation of amorphization is analyzed qualitatively by classical nucleation theory.
\end{abstract}

Key words: laser shock compression; silicon; amorphization; nanocrystalline; nano-twinning

*Corresponding author: Marc A. Meyers; Email: mameyers@eng.ucsd.edu 


\section{Introduction}

Silicon is an archetypal semiconductor with physical and chemical properties that continue to draw massive research interest. The mechanical behavior of silicon under quasi-static loading is well established as the result of several systematic investigations including mechanical testing and microstructural characterization [1,2]. Silicon is traditionally considered to be an ideally brittle material, lacking dislocation activity at room temperature [2]. It has a low fracture toughness that is comparable to ceramics [3] and shows considerable crystallographic anisotropy [4,5]. Silicon is also known to exhibit pressure-induced polymorphism and amorphization. Up to 13 different crystal structures of silicon have been reported among which the transition from diamond cubic to $\beta-\mathrm{Sn}$ at $10 \sim 12 \mathrm{GPa}$ is the most prominent [6-12]. Indentation and scratching investigations silicon reported near-surface amorphization [13-15], Gamero-Castaño and co-workers [16-18] have observed surface amorphization by nanodroplet impact and Deb et al. [19] compressed porous silicon film and identified pressure-induced amorphization. In addition to experimental studies, several thermodynamic and kinetic approaches have been implemented to study silicon amorphization mechanisms.[20-23] Demkowitz and Argon [24] performed MD simulations and predicted various amorphous silicon phases whose density depends largely on the cooling rate. Levitas [25] developed a kinetic and thermodynamic theory for strain-induced phase transitions, including amorphization, indicating that superposition of plastic work leads to a significant reduction in pressure required for strain induced chemical changes. Levitas [20] also proposed a virtual melting mechanism for crystalline to disordered transition.

Unlike silicon's quasi-static mechanical response, our understanding of silicon's dynamic behavior is still immature. Room temperature brittleness makes it experimentally difficult to examine its response under shock conditions and complicates post-mortem microscopy if the sample survives. For these reasons, reports on shock behavior of silicon are scarce and sometimes contradictory: Loveridge-Smith et al. [26] reported that silicon has an abnormally high Hugoniot elastic limit (HEL) when subjected to high amplitude pulsed laser shock, whereas Smith et al. [27] 
found inhomogeneous plastic flow, using a similar technique under the same relative conditions. There is also research indicating that silicon undergoes one of several phase transitions at equivalent pressures [28,29]. The large discrepancy of experimental results as well as computational simulations begs the question: what does silicon look like under shock loading? To definitively answer this question, two requirements are needed: successful recovery of shocked silicon samples experiment and informed computational simulation of the events connecting preand post-mortem characterization.

In a preceding letter [30], we reported that laser shock, at a strain rate of $\sim 10^{7} \mathrm{~s}^{-1}$, induced amorphization in monocrystalline silicon. A bulk amorphous surface layer and amorphous bands along favorable crystallographic directions were observed, both under transmission electron microscope (TEM) and in molecular dynamics (MD) simulation. It was proposed that large shear stress coupled with high pressure leads to the amorphization. In this investigation, we address this phenomenon, analyzing it quantitatively using thermodynamic parameters. Additionally, we evaluate the crystallization process from the amorphous state.

\section{Experimental Procedure}

\subsection{Laser Compression Experiment}

Laser compression experiments were carried out at Omega Laser Facility, Laboratory of Laser Energetics, University of Rochester. A pulsed neodymium glass laser with a wavelength of $351 \mathrm{~nm}$ was used. The full width half maximum pulse duration was $1 \mathrm{~ns}$ and nominal laser energies were $20,50,100,150,200$, and $450 \mathrm{~J}$. The lasers have a spot size of $3 \mathrm{~mm}$ and no phase plates were used in order to maximize the exposure area on the target. The target was assembled in a vacuum chamber and was pumped down to a pressure of $10^{-3} \mathrm{~Pa}$ during working conditions to prevent oxidation of the target and prevent absorption/scattering of the laser.

Silicon [001] single crystal 3mm x 3mm cylinders, purchased from Universitywafer LLC., were encapsulated in aluminum cups in order to protect the target from shattering. The close 
acoustic impedance of aluminum $(17.33 \mathrm{MPa} \cdot \mathrm{s} / \mathrm{m})$ to silicon $(19.7 \mathrm{MPa} \cdot \mathrm{s} / \mathrm{m})$ serves to minimize reflection of shock waves at interfaces/free surfaces, reducing damage and aiding successful recovery. Additionally, a $1 \mathrm{~mm}$ thick momentum trap was used to trap the shock wave at the rear surface. A dimensioned schematic of the target assembly is shown in Fig. 1.

The pulsed laser impacts the surface of the $20 \mu \mathrm{m}$ thick polystyrene $(\mathrm{CH})$ ablator turning the polymer into a plasma. The rapidly expanding plasma subsequently launches a planar shock wave into the $100 \mu \mathrm{m}$ thick aluminum "piston". The compression wave decays as it traverses the aluminum, ultimately arriving at the silicon target surface. The stress pulse profiles were simulated using HYADES, a 1-D hydrodynamic code. Peak pressure as a function of laser energy is shown in bottom-right panel of Fig. 1. For clarity, targets recovered from laser shock compression are denoted by their nominal laser energy followed by "shocked", e.g. $20 \mathrm{~J}$ shocked, $50 \mathrm{~J}$ shocked, etc.

\subsection{Microstructural Characterization}

Post-shock multi-scale microstructure analyses were carried out using different techniques. Scanning electron microscopy was used to characterize the surface morphology of the as-shocked target. Raman spectroscopy was applied to identify the existence of amorphous silicon in bulk regions. Transmission electron microscopy (TEM) and high resolution TEM (HRTEM) were used to characterize the microstructure evolution as a function of depth along the shock direction.

\subsubsection{Raman Spectroscopy}

Raman spectroscopy is considered to be a powerful tool to indicate vibrational, rotational, and other low-frequency modes in materials [31]. Thus, it is an extremely useful tool to distinguish between amorphous and crystalline phases. A cooled Princeton Instruments CCD detector equipped with a Spex $270 \mathrm{M}$ spectrometer was used to obtain Raman spectra on the as-shocked silicon targets. The specimens were mounted under a Nikon Optiphot microscope. Laser illumination was performed by focusing a $0.3 \mathrm{~kW} / \mathrm{cm}^{2}, 532 \mathrm{~nm}$ (wavelength) argon ion laser beam onto the top surface of specimens (adjacent to the area where TEM foils were extracted). The penetration depth of the illumination laser is approximately $0.5 \mu \mathrm{m}$. 


\subsubsection{TEM Sample Preparation}

The focused ion beam (FIB) technique was used to cut TEM foils directly from the as-shocked surface. For consistency and comparison, TEM foils of equivalent orientation are preferred. This is achieved by aligning the FIB cutting direction with the crack pattern observed on the surface plane. TEM foils were prepared in Oak Ridge National Laboratory using a Hitachi NB5000 scanning electron microscope with a dual beam FIB apparatus to cut TEM samples directly from the laser-shocked silicon monocrystal surface. The TEM foils were ion milled by 30 $\mathrm{kV} \mathrm{Ga}$ beam and finally polished at $5 \mathrm{kV}$ to minimize FIB damage. Before cutting the sample, the area of interest was aligned with the micro-crack network, as shown in Fig. 2. These cracks, orientated in $[110]$ and $[1 \overline{1} 0]$ directions, are most likely the traces of $\{111\}$ and/or $\{110\}$ cleavage planes. Three foils were prepared for each target in order to ensure the consistency of the results. Zero tilt electron diffraction patterns of all the samples are always within $\sim 2^{\circ}$ to the $\{110\}$ zone, indicating that the foil normal is $\langle 110\rangle$.

\subsubsection{Molecular Dynamics Simulation}

Simulations were accomplished with the LAMMPS package [32] utilizing a modified Tersoff interatomic potential [33] previously shown to have acceptable transferability to high pressure regimes[30]. Shock conditions are generated via infinite piston impact at a given particle velocity[34]. An impact orientation of [001] was selected for consistency with experimental work and transverse directions ([010] and [001]) have periodic boundary conditions applied. All MD snapshots were visualized using OVITO [35].

Our simulations are carried out with the MOD interatomic potential [33], which predicts a melting $\mathrm{T}$ of $1680 \mathrm{~K}$ at P=0 GPa. Simulations by Lane and coworkers [36] display completely elastic behavior for [001] propagation in a perfect crystal up to $\sim 32 \mathrm{GPa}$, with a relatively small temperature increase. Some amorphization can be observed at high pressures when nanovoids are added to the sample as pre-existing porosity. Recent simulations by Mogni et al. [29] report shock

melting of single crystal Si starting above $35 \mathrm{GPa}$ due to the nature of the modified Tersoff 
potential they used, which likely overestimates melting temperature.

\section{Results and Discussion}

The successful recovery of silicon from high shock pressures enabled subsequent microstructure characterization. In order to make the analysis consistent, all the TEM images were taken from the [110] zone axis and arranged in a way such that the shock wave travels from left to right $($ shock direction $=[001])$, unless noted otherwise .

\subsection{Observation of Shock Induced Amorphization}

\subsubsection{Raman Spectra}

Post-shock Raman spectroscopy was carried out to identify the amorphous silicon at a macroscopic scale. Fig. 2 illustrates Raman spectra as a function of laser energy. Beyond a laser energy threshold of $50.4 \mathrm{~J}$ (shock pressure threshold of $11.2 \mathrm{GPa}$ ), characteristics of amorphous silicon are observed. Both unshocked and $20 \mathrm{~J}$ shocked silicon targets show a sharp Raman peak at $520 \mathrm{~cm}^{-1}$ owing to the transverse optical (TO) phonon band, a characteristic of diamond cubic silicon. $50.4 \mathrm{~J}$ shocked targets shows an extra broad peak at $460-480 \mathrm{~cm}^{-1}$ that is attributed to the amorphous silicon (TO mode [37]) whereas the crystalline $520 \mathrm{~cm}^{-1}$ peak still exists due to the small thickness of amorphous layer. When laser energy increases to $106.8 \mathrm{~J}$, the $520 \mathrm{~cm}^{-1}$ peak vanishes and the $480 \mathrm{~cm}^{-1}$ amorphous peak dominates due to the increasing thickness of the amorphous layer $(\sim 2 \mu \mathrm{m}$, see TEM image Fig. 3 (a)) exceeding the penetration depth of the illumination laser. The spectrum of the $150 \mathrm{~J}$ shocked target shows a single $520 \mathrm{~cm}^{-1}$ shift due to the full crystallization at the top surface. It is also noted that the $520 \mathrm{~cm}^{-1}$ Raman peak for the 150 $\mathrm{J}$ shocked sample is broadened and shows an asymmetrical configuration compared with the $18.2 \mathrm{~J}$ shocked and unshocked sample. This is due to the presence of ultrafine grained/nanocrystalline materials[38] as presented later in Figure 9.

\subsubsection{TEM Observation}

In order to probe the microstructure of the shocked silicon, both conventional and high 
resolution TEM observations were carried out. They show that the crystal-to-amorphous transformation proceeds via a clear sequence of events. The recovered samples show several morphological configurations:

(a) Bulk transformation regions close to surface.

(b) Transformed bands that decrease in thickness with distance from the surface.

(c) Small 'feathering' features originating at the bands.

(d) Transformed regions at intersections of bands.

These different morphologies are shown in Fig. 3 and marked A,B,C,D, and E respectively.

Profuse stacking faults (SFs) and nanotwins are usually found in the vicinity of the amorphous bands, as imaged by HRTEM. These planar defects occur preferentially on $\{111\}$ planes. Fig 4 (a) shows such features with the apparent zigzag displacement highlighted in Fig. 4 (b). The thickness of each planar defect varied from 0.6 to $1 \mathrm{~nm}$, corresponding to $2 \sim 3$ atomic planes. Figs. 4 (c) and (d) show that SFs often intersect to create localized regions of high strain energy. Although these faults occur on $\{111\},\{112\}$ is also a favored twinning plane in silicon [39]. These faults act as precursors and favored nucleation sites for amorphization.

The formation of 'feathers' emanating from bands is best explained by Figure 5, which shows a [001] stereographic projection. The maximum shear stress cone is marked by a red dashed circle. The maximum shear stress occurs at an angle of $45^{\circ}$ to the shock wave propagation direction. The $\{111\}$ slip planes are also marked in the stereographic projection (green spots) and only coincide with the maximum shear cone at the orientations $[011],[101],[0 \overline{1} 1]$ and $[\overline{1} 01]$. For other orientations, and specifically for the one from which the current samples were extracted (plane normal $=[110])$ the angle of $(111)$ with $(001)$ is $54^{\circ}$. For this orientation, the (112) plane (blue spots) makes an angle of $35.3^{\circ}$ with the shock propagation direction ([001]). The maximum shear cone straddles these two planes, each $9^{\circ}$ away from the maximum in opposite directions. Thus, the resolved shear stresses on the (112) and (111) planes are identical. 
The current results indicate that although amorphization can initially take place along $\{111\}$, further propagation and growth almost always deviates a few degrees from $\{111\}$ towards the cone of maximum shear, as evidenced in Fig. 6.

\subsubsection{Molecular Dynamics Simulations}

Figure 7 shows a snapshot of the $14.5 \mathrm{GPa}$ shock. Three views are illustrated: a perspective view of defective atoms, a [110] projection of defective atoms, and a [110] projection of all atoms. "Defective" atoms are those filtered by deviating from four-fold coordination, with a cut-off of 0.3 nm. The third view (section through simulation box) is directly comparable to TEM analysis, while the first and second views illustrate the three-dimensional nature of the defective structures. Both single stacking faults and massive stacking-fault bands are observed. The massive stacking fault bands are comprised of successive stacking-faults layers, each occupying two atomic layers in the diamond cubic unit cell, for a stacking fault thickness of $\sim a_{0} / 2$. The majority of the massive stacking fault bands are comprised of $\{111\}$ stacking faults that make an angle of $54^{\circ}$ with [110] as compared to the $45^{\circ}$ cone of maximum shear. A tendency towards deforming along the direction of maximum shear manifests itself as subsequent stacking-fault steps inclined at an angle of $9^{\circ}=54^{\circ}-45^{\circ}$ and result in lengths between steps of $\sim 1.7 \mathrm{~nm}$ as illustrated in Figure 8 . As compression increases up to a strain of 0.15 , the $\{111\}$ plane can deviate up to $5^{\circ}$ from its original position, further causing resultant amorphous bands to vary in angle.

\subsection{Mechanisms of Shock induced Amorphization}

The crystalline-to-amorphous transition through application of pressure (and/or shear) has been a subject of intense study for the past decades[40], ever since the seminal discovery of pressure-induced amorphization in ice by Mishima et al.[41]in 1984. A similar phenomenon was found in silica [42], boron carbide[43], tantalum oxide[44], porous silicon thin films[19], and many other materials systems[45]. Under shock compression, concomitant high pressure and shear stresses were postulated to be responsible for the amorphization of silicon[30]. In the following sections, mechanisms of amorphization are proposed. 


\subsubsection{Microstructural Considerations}

The onset of amorphization is strongly affected by shear stresses and deformation. All evidence indicates that the transformation is preceded by twins/stacking faults; these defects advance with the shock wave and promote amorphization.

Figure 9 shows in schematic fashion how these defects can give rise to amorphized regions that can reorient themselves to seek other directions where their propagation is favored. A set of (1-11) SFs is generated under an applied stress and penetrates into the sample. These stacking faults serve as the nucleation sites for amorphization, as shown in Fig. 9(b). Once the amorphous nucleus is formed, its growth tends to deviate towards the orientation of the maximum shear (Fig. 9 (c)). Furthermore, bifurcation of the primary bands into $(1 \overline{1} \overline{2})$ is shown in Fig. 9 (d).

There is also evidence, shown in Fig. 4 (c), of nucleation of amorphized regions at intersections of twin/stacking faults. Stacking-fault intersections are regions of higher energy because of the additive nature of elastic strain. Indeed, Lagneborg [46] observed the nucleation of martensite at twin-twin and $\varepsilon-\varepsilon$ intersections. A similar mechanism is proposed in Figure 10, which shows two intersecting groups of stacking-faults/twins giving rise to the initiation of amorphization.

The evolution of defects and their role in amorphization was informed by molecular dynamics simulations. Fig. 11 exemplifies the three modes of amorphization discussed thus far: bulk surface amorphization, amorphization along bands of stacking faults, and amorphization at stacking fault intersections. The bulk disordered layer at the surface is not observed in simulations where the piston is directly bonded to the sample, since surface defects/roughness play an important role in this phenomenon. At $\mathrm{t}=8 \mathrm{ps}$ we see the formation of a disordered band within the stacking fault band at the bottom of the frame. At $\mathrm{t}=10 \mathrm{ps}$ the first intersection of stacking faults takes place and at $\mathrm{t}=13 \mathrm{ps}$ an amorphous nucleus is formed at this intersection while others intersect with a greater number of stacking faults.

\subsubsection{Thermodynamic Analysis}


Assuming that amorphous $\mathrm{Si}$ is energetically equivalent to liquid $\mathrm{Si}$, i.e. that their Gibbs free energy curves are identical, amorphization can be treated as melting at lower temperatures as a first approximation.

(i) Amorphization Energetics Fig. 12 (a) shows the Gibbs free energies (energy per unit volume) of amorphous silicon (a-Si) and crystalline silicon (c-Si) as function of temperature in the absence of applied stress[47]. The intersection gives the melting temperature where the Gibbs free energy of a-Si equals that of c-Si. At lower temperatures, c-Si is energetically more favorable and the transformation of c-Si into a-Si will lead to an energy increase, which serves as the energy barrier $\left(\Delta \mathrm{G}_{\mathrm{c}-\mathrm{a}}\right)$ of the $\mathrm{c}$-a transformation. Assuming the difference between Gibbs free energy curves to be linear, $\Delta \mathrm{G}_{\mathrm{c}-\mathrm{a}}$ can be expressed by:

$$
\Delta \mathrm{G}_{\mathrm{c}-\mathrm{a}}(\mathrm{T})=\Delta \mathrm{G}_{\mathrm{c}-\mathrm{a}}(300 \mathrm{~K})\left(1-\frac{\mathrm{T}}{\mathrm{T}_{\mathrm{m}}}\right)
$$

The green solid line in Fig. 12 (b) shows $\Delta \mathrm{G}_{\mathrm{c}-\mathrm{a}}$ as function of temperature (without the application of pressure and/or shear); At 300, $\mathrm{K}$ the value of $\Delta \mathrm{G}_{\mathrm{c}-\mathrm{a}} \approx 41 \mathrm{~kJ} / \mathrm{mol}$. The high energy barrier prevents the c-a transformation at lower temperatures. However, this may overcome by the assistance of external work. It has been shown that stress plays a very important role in solid state phase transformations [48-50]. Patel and Cohen [51] were the first to study the influence different stress states on the martensitic transformation under quasi-static loading and to propose a rationalization. Using the same formalism, Thadhani and Meyers [52] were able to evaluate the martensitic transformation under tensile pulses propitiated by reflected shock waves. This framework is applied here.

Analogous to the Patel and Cohen rationalization, the work $(W)$ done on the transformation due to the action of applied stress can be separated into two contributions: (1) a hydrostatic pressure term taken as the product of the hydrostatic stress and volumetric strain of the transformed region $\left(\mathrm{P} \varepsilon_{\mathrm{v}}\right)$, and (2) a shear energy term taken as the product of the deviatoric shear 
stress and shear strain producing $(\tau \gamma)$. Thus, the total work per unit volume is the sum of the two contributions,

$$
\mathrm{W}=\mathrm{P} \varepsilon_{\mathrm{v}}+\tau \gamma
$$

Under shock compression, the high amplitude of pressure and shear stress are coupled and applied simultaneously. The effect of shock compression is shown in Fig. 12 (b); the energy barrier shifts downwards due to the contribution of pressure and shear. A general relationship can be expressed as,

$$
\Delta \mathrm{G}_{\mathrm{c}-\mathrm{a}}(\mathrm{T} ; \mathrm{P}, \tau=0)=\mathrm{P} \varepsilon_{\mathrm{v}}+\tau \gamma+\Delta \mathrm{G}_{\mathrm{c}-\mathrm{a}}(\mathrm{T} ; \mathrm{P}, \tau)
$$

If $P \varepsilon_{v}+\tau \gamma$ is sufficiently high to exceed $\Delta \mathrm{G}_{\mathrm{c}-\mathrm{a}}(\mathrm{T} ; \mathrm{P}, \tau=0), \Delta \mathrm{G}_{\mathrm{c}-\mathrm{a}}(\mathrm{T} ; \mathrm{P}, \tau)$ can become zero or even negative, implying that the c-a transformation will occur spontaneously. The shock pressure, $\mathrm{P}_{\mathrm{zz}}$, hydrostatic pressure, $\mathrm{P}$, and maximum shear stress, $\tau_{\max }$ are related by[53],

$$
P_{\mathrm{zz}}=\mathrm{P}+\frac{4}{3} \tau_{\max }
$$

In elastic compression, the ratio of $\tau_{\max }$ over $\mathrm{P}$, for cubic materials loaded along [001] direction, is given as function of the elastic constants:

$$
\frac{\tau_{\max }}{P}=\frac{3\left(C_{11}-C_{12}\right)}{2\left(C_{11}+2 C_{12}\right)}
$$

$\mathrm{C}_{11}$ and $\mathrm{C}_{12}$ are pressure dependent stiffness, resulting in a pressure dependent relationship between shear stress and hydrostatic pressure. Molecular dynamics simulations using the MOD potential show good agreement with pressure dependent stiffness and predict $\frac{\tau_{\max }}{P}$ as a function of pressure. Shock pressures obtained from 1-D hydrodynamic simulations and corresponding $P$ and $\tau_{\max }$ are calculated and shown in the Table 1.

Transformation of c-Si to high density a-Si at elevated pressures leads to a volume shrinkage $\Delta V \cong 0.91 \mathrm{~cm}^{3} /$ mole, rendering $\varepsilon_{\mathrm{v}}=\frac{\Delta \mathrm{V}}{V}=0.075$ [19]. TEM observation shows that 
amorphization tends to occur in regions of massive stacking faults/nanotwins. Hence, $\gamma$ can be estimated, as a first approximation, by the characteristic twinning strain of 0.707 for cubic crystals. Thus, $W$, calculated by Eq. 2, increases monotonically with shock pressure. The compressive nature of shock loading favors such a c-a transformation by reducing the energy barrier, $\Delta \mathrm{G}_{\mathrm{c}-\mathrm{a}}$, as shown in Fig. 12(b), where the solid-dotted lines denote a reduced $\Delta \mathrm{G}_{\mathrm{c}-\mathrm{a}}$ for three different laser energies corresponding to different shock pressures. The intersections of the solid-dotted lines with the temperature axis indicate $\mathrm{c}$-a transformation temperatures at which $\Delta \mathrm{G}_{\mathrm{c}-\mathrm{a}}=0$. Fig. 12 (c) shows the effect of shock pressure on the c-a transformation temperature.

It should be noted that the temperature rise associated with shock can be calculated analytically by Eq. 6 [53],

$$
T=T_{0} \exp \left[\left(\frac{\gamma_{0}}{V_{0}}\right)\left(V_{o}-V\right)\right]+\frac{V_{o}-V}{2 C_{V}} P+\frac{\exp \left(-\frac{\gamma_{0}}{V_{o}} V\right)}{2 C_{V}} \int_{V_{0}}^{V} P \exp \left[\frac{\gamma_{0}}{V_{o}} V\right]\left[2-\frac{\gamma_{o}}{V_{o}}\left(V_{o}-V\right)\right] d V
$$

$\gamma_{0}$ is the Grüneisen parameter and $C_{v}$ is the heat capacity. The shock-induced temperature (shock temperature) rise was calculated in a previous paper [30] and is plotted in Fig. 12 (c) along with the c-a transformation temperature. The temperature rise at shock front will further facilitate the c-a transformation.

(ii) Nucleation The nucleation of amorphous phase occurs preferentially in connection with the stacking faulted regions. Note that nucleation of a-Si introduces extra interfaces which lead to energy increase, whereas this event also consumes the stacking faults, resulting in an energy decrease. Assuming an ellipsoidal nucleus with radius $r$ and semi-thickness $c$, the aspect ratio $f=\frac{c}{r}$ defines the shape of the nucleated embryo. Hence, the net Gibbs free energy gain of amorphization is:

$$
\Delta \mathrm{G}=\frac{4 \pi \mathrm{r}^{2} c}{3} \cdot \mathrm{G}_{\mathrm{c}-\mathrm{a}}+4 \pi \mathrm{rc} \gamma_{\mathrm{ac}}-\frac{4 \pi \mathrm{r}^{2} c}{3} \gamma_{\mathrm{SF}} \rho_{\mathrm{SF}}-\frac{4 \pi \mathrm{r}^{2} c}{3} \cdot \frac{1}{2} \rho_{\mathrm{d}} \mathrm{G}_{\{111\}} \mathrm{b}_{p}^{2}
$$

where $\gamma_{\mathrm{ac}}$ is the interfacial energy, $\gamma_{\mathrm{SF}}, \rho_{\mathrm{SF}}, \rho_{\mathrm{d}}$ denote stacking-fault energy, partial dislocation 
density, and dislocation density, respectively. $\mathrm{b}_{p}$ is the Burgers vector of the partial dislocations. $\mathrm{G}_{\{111\}}$ is the shear modulus of the materials on $\{111\}$ slip plane. $\frac{1}{2} \rho_{\mathrm{d}} \mathrm{G}_{\{111\}} \mathrm{b}_{p}{ }^{2}$ is approximately the stored elastic energy of dislocations per unit volume. TEM observations, as evidenced in Fig. 4, suggest that the amorphous structure tends to exhibit a more disc-like than spherical shape, i.e. $f<<1$. It should be mentioned that one stacking fault corresponds to two partial dislocations, rendering,

$$
\rho_{\mathrm{d}}=\frac{2 \rho_{\mathrm{SF}}}{1_{\mathrm{SF}}}
$$

Where $1_{\mathrm{SF}}$ is the width of stacking faults, i.e. the distance that a perfect dislocation dissociates. Substituting Eq. 8 into Eq. 7;

$$
\Delta \mathrm{G}=\frac{4 \pi \mathrm{r}^{3}}{3} \cdot f \cdot\left(\Delta \mathrm{G}_{\mathrm{c}-\mathrm{a}}-\gamma_{\mathrm{SF}} \rho_{\mathrm{SF}}-\frac{\rho_{\mathrm{SF}}}{1_{\mathrm{SF}}} \mathrm{G}_{\{111\}} \mathrm{b}_{p}{ }^{2}\right)+4 \pi \mathrm{r}^{2} \gamma_{\mathrm{ac}} \cdot f
$$

The $\Delta \mathrm{G}$ vs. $\mathrm{r}$ curve has three different configurations, depending on the sign of $\Delta \mathrm{G}_{\mathrm{c}-\mathrm{a}}-\gamma_{\mathrm{SF}} \rho_{\mathrm{SF}}-\frac{\rho_{\mathrm{SF}}}{1_{\mathrm{SF}}} \mathrm{G}_{\{111\}} \mathrm{b}_{p}^{2}:$

(1) If $\Delta \mathrm{G}_{\mathrm{c}-\mathrm{a}}-\gamma_{\mathrm{SF}} \rho_{\mathrm{SF}}-\frac{\rho_{\mathrm{SF}}}{1_{\mathrm{SF}}} \mathrm{G}_{\{11\}} \mathrm{b}_{p}{ }^{2}>0, \Delta \mathrm{G}$ is positive and increases monotonically with $\mathrm{r}$. The Gibbs free energy continues to increase once the amorphous nucleus is formed. Therefore, the solid state amorphization will not happen.

(2) If $\Delta \mathrm{G}_{\mathrm{c}-\mathrm{a}}-\gamma_{\mathrm{SF}} \rho_{\mathrm{SF}}-\frac{\rho_{\mathrm{SF}}}{1_{\mathrm{SF}}} \mathrm{G}_{\{111\}} \mathrm{b}_{p}{ }^{2}=0$, the $\mathrm{r}^{3}$ term on the right-hand side vanishes and the energy increase of the system is associated with the introduction of the new c-a interfaces. Therefore, the solid state amorphization is unfavorable.

(3) If $\Delta \mathrm{G}_{\mathrm{c}-\mathrm{a}}-\gamma_{\mathrm{SF}} \rho_{\mathrm{SF}}-\frac{\rho_{\mathrm{SF}}}{1_{\mathrm{SF}}} \mathrm{G}_{\{11\}\}} \mathrm{b}_{p}{ }^{2}<0, \Delta \mathrm{G}$ vs. $\mathrm{r}$ curve becomes convex with the 
maximum value at a critical radius. Therefore, once the amorphous nucleus exceeds the critical value, amorphization will take place spontaneously.

Considering $f$ to be constant, classical nucleation theory [54] gives the critical nucleus size and energy barrier for the condition $\frac{\partial \Delta \mathrm{G}}{\partial \mathrm{r}}=0$,

$$
\begin{gathered}
\mathrm{r}_{\mathrm{c}}=-\frac{2 \gamma_{\mathrm{ac}}}{\Delta \mathrm{G}_{\mathrm{c}-\mathrm{a}}-\gamma_{\mathrm{SF}} \rho_{\mathrm{SF}}-\frac{\rho_{\mathrm{SF}}}{1_{\mathrm{SF}}} \mathrm{Gb}_{p}{ }^{2}} \\
\text { and } \Delta \mathrm{G}_{\mathrm{c}}=f \cdot \frac{16 \pi \gamma_{\mathrm{ac}}{ }^{3}}{3\left(\Delta \mathrm{G}_{\mathrm{c}-\mathrm{a}}-\gamma_{\mathrm{SF}} \rho_{\mathrm{SF}}-\frac{\rho_{\mathrm{SF}}}{1_{\mathrm{SF}}} \mathrm{Gb}_{p}{ }^{2}\right)^{2}}
\end{gathered}
$$

$\Delta \mathrm{G}_{\mathrm{c}-\mathrm{a}}-\gamma_{\mathrm{SF}} \rho_{\mathrm{SF}}-\frac{\rho_{\mathrm{SF}}}{1_{\mathrm{SF}}} \mathrm{G}_{\{111\}} \mathrm{b}_{p}{ }^{2}=0$ gives a critical value of the stacking fault density:

$$
\rho_{\mathrm{SF}}=\frac{\Delta G_{\mathrm{c}-\mathrm{a}}}{\gamma_{\mathrm{SF}}+\frac{\mathrm{G}_{\{111\}} b^{2}}{1_{\mathrm{SF}}}}
$$

Assuming a linear isotropic elastic medium, $1_{\mathrm{SF}}$ can be estimated by[55]

$$
1_{\mathrm{SF}}=\frac{G_{\{111\}} b_{p}^{2}}{8 \pi \gamma_{S F}} \cdot \frac{2-v}{1-v} \cdot\left(1-\frac{2 v \cdot \cos 2 \Theta}{2-v}\right)
$$

Where $v$ is the Poisson ratio and $\Theta$ is the angle between Burgers vector and line element of the perfect dislocation. For cubic crystals[56,57],

$$
G_{\{111\}}=\frac{3 C_{44}\left(C_{11}-C_{12}\right)}{4 C_{44}+C_{11}+C_{12}}
$$

For silicon, $\Theta$ is $60^{\circ} . v=0.215[58], \gamma_{S F} \approx 55 \mathrm{~mJ} / \mathrm{m}^{2}[59]$, and $G_{\{111\}} \approx 44.3 G P a[60]$ and $\gamma_{a c} \approx \gamma_{c l}=0.416 J / m^{2}$ [61]. At $300 \mathrm{~K}, \Delta \mathrm{G}_{\mathrm{c}-\mathrm{a}}=41 \mathrm{~kJ} / \mathrm{mol}$ [47], renders a critical stacking fault density of $\rho_{S F}=5.7 \times 10^{9} \mathrm{~m}^{-1}$ which corresponds to a critical dislocation density of 
$\left.\rho_{d}\right|_{P z z=0}=2.84 \times 10^{18} \mathrm{~m}^{-2}=2.84 \times 10^{14} \mathrm{~cm}^{-2}$. Thus, an extremely high density of stacking faults/dislocations is required to compensate for the energy gain of c-a transformation at room temperature, making the c-a transformation impossible.

Under shock compression, as shown in Fig. 12, $\Delta \mathrm{G}_{\mathrm{c}-\mathrm{a}}$ decreases as pressure and shear stress increases. Additionally, the temperature rise at the shock front further decreases the energy barrier. Correspondingly, the required dislocation density to trigger c-a transformation is also reduced. For instance, at a shock pressure of $11 \mathrm{GPa}\left(E_{\text {laser }}=50.4 \mathrm{~J}\right), \Delta \mathrm{G}_{\mathrm{c}-\mathrm{a}} \approx 5 \mathrm{~kJ} / \mathrm{mol}$ at room temperature (as shown in Fig. 12 (b)), and Eq. 12 gives the required $\left.\rho_{d}\right|_{P z z=11 G P a} \approx 3.5 \times 10^{13} \mathrm{~cm}^{-2}$, which is on the same order of magnitude of the defect density as value measured from HRTEM. This explains why amorphization usually initiates either along stacking faults packets or their intersections, as proposed in the previous section. Figure 13 illustrates the effect of defect (partial dislocation) density on the nucleation energy of an amorphous embryo under shock compression. The aspect ratio of the amorphous nuclei is assumed to be constant and $f=0.01$ is taken as a first approximation. Below the critical dislocation density of $3.5 \times 10^{13} \mathrm{~cm}^{-2}$ (dark green), nucleation of amorphous structure will always result in an energy increase. Beyond this critical condition, further increase of dislocation density enables amorphization.

Under strong shock compression, $\Delta \mathrm{G}_{\mathrm{c}-\mathrm{a}}$ may become negative at a sufficient pressure. This is the case of $106.8 \mathrm{~J}$; no lattice defects are required for amorphization to occur. This is supported by a bulk layer of amorphous structure without lattice defects at c-a interfaces. However, as the shock wave propagates through the material, it is rapidly attenuated and therefore $\Delta \mathrm{G}_{\mathrm{c}-\mathrm{a}}$ increases. Thus, a transition of bulk amorphization to directional amorphization can be expected. In the latter case, shock-induced defect generation plays a crucial role.

The dislocation density at the shock front can be calculated from a homogeneous nucleation mechanism[62], 


$$
\rho_{d}=\frac{2 \pi^{2}}{0.8(1-v) k^{3} b_{0}^{2}}\left(\frac{V}{V_{0}}\right)^{-2 / 3}\left[1-\left(\frac{V}{V_{0}}\right)^{1 / 3}\right]^{3}
$$

In which $\mathrm{k}$ is the orientation factor, $\mathrm{b}_{0}$ is the Burgers vector $\mathrm{V}$ is the compressed specific volume and $\mathrm{V}_{0}$ is the specific volume in the relaxed state. The specific volume can be related to shock pressure by Rankine-Hugoniot relationship[53],

$$
P_{z z}=\frac{\mathrm{C}_{0}^{2}\left(1-\frac{V}{V_{0}}\right)}{V_{0}\left[1-S\left(1-\frac{V}{V_{0}}\right)\right]^{2}}
$$

Where $\mathrm{S}$ and $\mathrm{C}_{0}$ are experimentally-determined parameters characteristic of materials $\left(U_{s}=C_{0}+S U_{p}\right)$. Therefore, the shock-induced dislocation density can be expressed as function of shock pressure, as shown in Figure 14. It can be inferred that $\rho_{d} \approx 1 \times 10^{12} \mathrm{~cm}^{-2}$ when $P_{z z}=11 G P a\left(E_{l a s e r}=50.4 \mathrm{~J}\right)$, which matches reasonably well the previously calculated critical dislocation density of $\left.\rho_{d}\right|_{P_{Z z}=1 G P a} \approx 3.5 \times 10^{13} \mathrm{~cm}^{-2}$ for amorphization to be initiated at this shock energy level. We note that Eq. 15 gives the "global" dislocation density produced by the shock, and that MD simulated dislocation densities in the localized plastic regions are much higher and extremely close to the critical value estimated above for amorphization.

It should be noted that shock-induced defects also affect the activation energy $\left(\Delta \mathrm{G}_{\mathrm{c}}\right)$ to form a critical nucleus size, i.e. the higher the defect density, the lower $\Delta G_{c}$, as shown in Figure 13. Under medium to high amplitude shock compression, the high defect density and associated heat might enable thermally-activated nucleation. However, the fast kinetics of laser shock compression favors nucleation controlled by strain rather than time. Thus the barrier-less nucleation proposed by Levitas $[25,63]$ is very likely to occur. The amorphous silicon thus formed is most likely of high density (liquid-like), which is also the carrier of plasticity for amorphous silicon, according to Demkowicz and Argon [24]. 


\subsection{Nanocrystallization of a-Si}

As laser energy increases, so does the heating effect of shock. Since the amorphous structure is thermodynamically metastable, it can transform into a crystalline structure, upon unloading. Indeed this was observed in the high energy $(150 \mathrm{~J})$ experiment and is analyzed in this section.

\subsubsection{Grain Size Gradient Along the Shock Direction}

As shown in Fig. 15, the $150 \mathrm{~J}$ shocked sample exhibits a polycrystalline microstructure close to the shock surface, whereas amorphous bands were still seen $10 \sim 12 \mu \mathrm{m}$ below the shock surface. The sharp diffraction ring confirms the polycrystalline nature; the grains are equiaxed. Grain-size distribution was measured by the line intersection method as a function of depth along the shock direction. A grain size gradient emerges with coarse grains on the surface and finer grains within. Depending on the grain sizes, as shown in Fig. 16, four different regions can be delineated, namely, (1) coarse grain silicon (CG-Si) with a grain size around $1 \mu \mathrm{m}$ (correspond to Fig. 15a); (2) ultrafine grained silicon (UFG-Si) with a grain size of 150 $\pm 60 \mathrm{~nm}$ (Fig. 15b); (3) nanocrystalline silicon (nc-Si) with a grain size of $50 \pm 20 \mathrm{~nm}$ (Fig. 15c); and (4) a mixture of amorphous and monocrystalline silicon (a/m-Si, Fig. 15d). The boundaries between different regions, however, are not well defined and some large grains, possibly due to abnormal grain growth can be observed in the nanocrystalline region. It is also interesting to see a high density of twin structures with nanometer thickness in UFG-Si and nc-Si. Also, various contrasted spots were found on the contrast-free residual amorphous domains, indicating crystalline structures within the amorphous silicon.

The amorphous phase can transform into nanocrystalline silicon through two possible mechanisms: (1) crystallization from the molten phase; (2) crystallization directly from the amorphous phase. TEM observations (Fig. 15d) seem to favor the second mechanism since multiple crystalline "islands" can be identified on the preserved amorphous bands, indicating an early stage of nucleation. 


\subsubsection{Crystallization Mechanism}

Crystallization from amorphous materials is akin to recrystallization from heavily cold-deformed metals and alloys; however, they differ in driving force, i.e. in the former, crystallization is driven by the Gibbs free energy difference between amorphous and crystalline states whereas, in the later, recrystallization is promoted by the stored elastic energy due to previously imposed cold work. Despite this difference, they share common foundations: both are based in nucleation and growth. In light of this, three stages of crystallization can be expected: (1) formation of nuclei with a critical size; (2) grain growth at expense of the surrounding amorphous materials; (3) grain impingement and continuous grain growth via motion of high-angle grain boundaries.

The driving force of crystallization is the Gibbs free energy difference between a-Si and c-Si, $\Delta G_{a-c}(T)=g_{a}(T)-g_{c}(T)$. Considering a homogeneous nucleation mechanism and spherical nuclei, one has,

$$
r_{c}=\frac{2 \gamma_{c-a}}{\Delta G_{a-c}}
$$

$\Delta G_{a-c}\left(\frac{T_{m}}{2}\right) \approx 1.9 \mathrm{GPa}(23.2 \mathrm{~kJ} / \mathrm{mol})$ can be obtained from Fig. $12(\mathrm{~b})$, if it is assumed that $\frac{T_{m}}{2}$

the crystallization temperature. This is two orders of magnitude higher than the driving force for recrystallization for cold-deformed metals, which is usually on the order of $10 \mathrm{MPa}(0.12 \mathrm{~kJ} / \mathrm{mol})$ $[64,65] . \gamma_{c-a}$ is the same for crystallization as that for amorphization and has a reported range of $0.4 \sim 2 \mathrm{~J} / \mathrm{m}^{2}[61,66-68]$, rendering a critical grain size of $D_{c}=2 r_{c}=0.4 \sim 2 \mathrm{~nm}$.

Figure 17 (a) displays a spherical crystalline nucleus $(D \sim 10 \mathrm{~nm})$ embedded in an amorphous matrix. A magnified view (Fig. 17(b)) of the area enclosed by the box in Fig. 17 (a) clearly shows the twinning structure,. These are the characteristic growth twins, and not deformation twins. Note that the intersection of the mirror plane with the c/a interface forms a triple junction, which influences the grain growth kinetics. 
As the crystallization proceeds, the volume fraction of the amorphous material shrinks, resulting in decrease number of nucleation sites. Nucleation ceases once all the amorphous phase is consumed. After this, grains start to impinge on each other, leading to faceting of the spherical interfaces. Grain impingement also leads to the formation of grain boundaries and the motion of which generates the further grain growth [69].

The crystallized microstructure is influenced by nucleation rate, $\dot{N}$, and grain growth rate,

$\frac{d r}{d t}$. The competition between the two factors determines the final grain size, i.e. the larger $\dot{N}$ and the smaller $\frac{d r}{d t}$, the finer the grain size. The complete randomness of amorphous structure provides plentiful nucleation sites of equal probability. Nucleation at such small critical sizes is readily achievable by thermal fluctuation and thus it is preferred over grain growth before grain impingement, resulting in a very fine grain size. The boundaries between three stages of the crystallization are difficult to delineate. However, since the critical size for homogeneous nucleation is small and the temperature at this stage is sufficiently high, it is postulated that nucleation has fast kinetics and therefore the grain growth after grain impingement is the rate-controlling step.

\subsubsection{Influence of triple junctions on grain growth}

The specific kinetic and thermodynamic properties of triple junctions strongly impact the microstructure evolution of polycrystals [70]. Gottstein and Schvindlerman [70,71] demonstrated that triple junctions drag the motion of grain boundaries and therefore influence the kinetics of grain growth. Such a phenomenon is especially important when the grain size is in nano-scale.

It is proposed here that triple junctions may play a very important role in the nanocrystallization of amorphous silicon. As mentioned above, nuclei are distributed homogeneously in the amorphous matrix; therefore, numerous triple junctions can be expected. Considering the triple junction effect on the grain growth, one has the modified Nernst-Einstein equation [71], 


$$
\frac{d r}{d t}=\frac{m_{b} F}{\left(1+\frac{1}{\wedge_{t j}}\right)}
$$

Where $r$ is the grain radius, $m_{b}$ is the mobility of grain boundary and/or c-a interface, $\wedge_{t j}=\frac{m_{t j}}{m_{b}} \cdot D$ is the dimensionless parameter related to the mobility of triple junction $\left(m_{t j}\right)$ and $m_{b}$. The driving force of the grain growth is:

$$
F=\frac{2 \gamma_{g b}}{r}=\frac{4 \gamma_{g b}}{D}
$$

$D=2 r$ is the average grain size.

If $\wedge_{t j}>>1$, grain-boundary mechanism is the controlling process of grain growth kinetics and $D \sim t^{0.5}$. This is similar to the Hu-Rath equation [72] that was used by Lu et al.[73]

If $\wedge_{t j}<<1$, the triple junction is the dominant factor for grain growth kinetics and $D \sim t$. One should note that the triple lines in polycrystalline materials not only retard the grain growth by dragging the motion of the grain boundary but also provide extra driving force $F_{t j}=\frac{36 \gamma_{t j}}{\pi r^{2}}[74,75]$ where $\gamma_{t j}$ is the triple line tension. $F=F_{t j}$ gives a critical grain size $D_{c}$ at which the driving force contributed by grain boundary equals. The triple line. The triple line energy of silicon has not been measured experimentally whereas MD simulation gives a value of $8.6 \times 10^{-8} \mathrm{~J} / \mathrm{m}[67]$. The grain boundary energy is measured to be $0.45 \sim 0.5 \mathrm{~J} / \mathrm{m}^{2}$ [66]. Therefore, a critical diameter, $D_{c} \approx 22 \mathrm{~nm}$ is obtained. Thus, the effect of triple line on the grain growth kinetics should be taken into account at least until the grain size reaches $22 \mathrm{~nm}$; this is especially important after the nucleating grains encounter.

Kinetics of melting and crystallization has been studied for a variety of systems $[61,76]$. The pressure effect on the crystallization from amorphous phase was first proposed by Ye and Lu [77]. Recently, high pressure melting and crystallization into a nanocrystalline structure was 
simulated for Ta [78], and it was shown that classical nucleation theory can provide a reasonable picture of the crystallization process. Under shock compression, the crystallization will most likely occur during the unloading path due to the rapid decay of the pressure pulse, since the crystalline form is stable at room temperature and pressure.

\section{Conclusions}

It is established that high-power, short-duration, laser-driven shock compression of silicon single crystals leads to amorphization. The principal conclusions are summarized as:

(1) The amorphization occurs above a threshold shock pressure. The amorphous material is composed of a surface layer and multiple bands that propagate into the crystal. The thickness of the amorphous layer and bands increases with laser energy and decreases with depth below the shock surface.

(2) TEM observation revealed that these amorphous bands tend to show a specific crystallographic orientation, i.e. they align with $\{111\}$ slip planes (up to $\sim 10^{\circ}$ of deviation was observed). Large numbers of bifurcations were found on the sides of the amorphous bands. HRTEM shows abundant stacking faults in the vicinity of the amorphous band, suggesting that shock-induced lattice defects are precursors to amorphous band formation. Deviation of amorphous bands from $\{111\}$ slip planes can be explained by their tendency to align themselves along maximum shear directions.

(3) Large scale MD simulations show shock-induced amorphization with patterns which agree well with TEM/HRTEM observations. Simulations display nucleation and growth of SFs, which can then intersect. Amorphization starts alongside these SFs and also at their intersection within ps of plasticity initiation.

(4) Following the Patel and Cohen[51] formalism, the energetics of shock-induced amorphization was analyzed. At a temperature below melting temperature, the c-a transformation has to overcome a high energy barrier, rendering it impossible to occur under ambient condition. Under shock compression, however, the high magnitude of the coupled hydrostatic pressure and 
associated deviatoric component dramatically lowers the energy barrier, rendering the c-a transformation possible. The temperature rise at the shock front further facilitates amorphization. (5) The heterogeneous nucleation of the amorphous phase from the highly dislocated structures is proposed and it is shown that partial dislocations and stacking fault packets and their intersections give rise to nucleation.

(6) At the highest laser energy reported here, recrystallization of amorphous silicon into its nanocrystalline counterpart was documented. This is due to thermodynamic driving forces, i.e. the Gibbs free energy of amorphous silicon being higher than that of crystalline silicon.

(7) A grain size gradient was observed from the shock surface towards the interior of the sample; the size decreased from $1 \mu \mathrm{m}$ at the shock surface to tens of nanometers a few $\mu \mathrm{m}$ below. Extensive annealing twinning was observed within the nanograins. Thus, it is proposed that the crystallization occurs upon unloading, after the passage of the shock pulse.

\section{Acknowledgement}

This research is funded by a UC Research Laboratories Grant (09-LR-06-118456-MEYM) and a National Laser Users Facility (NLUF) Grant (PE-FG52-09NA-29043). We acknowledge the highly professional support of the LLE Omega laser facility and supporting staff in addition to Tane Remington for target assembly. Electron microscopy was conducted at ShaRE (CNMS) User Facility, Oak Ridge National Laboratory, which is sponsored by Office of Basic Energy Science, US. Department of Energy. We thank Dorothy Coffey for assistance with the FIB sample preparation. Computational resources from Los Alamos National Laboratory were supported by DOE Office of Science, Office of Advanced Scientific Computing (ASCR) via the Exascale Co-design Center for Materials in Extreme Environments. EMB thanks support from pict0092 and a SeCTyP grant. 


\section{References}

[1] G.M. Pharr, W.C. Oliver, D.R. Clarke, The mechanical behavior of silicon during small-scale indentation, J. Electron. Mater. 19 (1990) 881-887. doi:10.1007/BF02652912.

[2] J. Rabier, L. Pizzagalli, J.L. Demenet, Dislocations in Silicon at High Stress, Elsevier, 2010. doi:10.1016/S1572-4859(09)01602-7.

[3] M.A. Meyers, K.K. Chawla, Mechanical Behavior of Materials, 2nd ed., Cambridge University Press, 2008.

[4] R. Perez, P. Gumbsch, Directional anisotropy in the cleavage fracture of silicon, Phys. Rev. Lett. 84 (2000) 5347-50. http://www.ncbi.nlm.nih.gov/pubmed/10990940.

[5] D.R. Clarke, Fracture of Silicon and Other Semiconductors, 1992.

[6] A. Mujica, A. Rubio, A. Muñoz, R. Needs, High-pressure phases of group-IV, III-V, and II-VI compounds, Rev. Mod. Phys. 75 (2003) 863-912. doi:10.1103/RevModPhys.75.863.

[7] M.C. Gupta, A.L. Ruoff, Static compression of silicon in the [100] and in the [111] directions, J. Appl. Phys. 51 (1980) 1072. doi:10.1063/1.327714.

[8] J. Yan, H. Takahashi, X. Gai, H. Harada, J. Tamaki, T. Kuriyagawa, Load effects on the phase transformation of single-crystal silicon during nanoindentation tests, Mater. Sci. Eng. A. 423 (2006) 19-23. doi:10.1016/j.msea.2005.09.120.

[9] J. Jang, M.J. Lance, S. Wen, T.Y. Tsui, G.M. Pharr, Indentation-induced phase transformations in silicon: influences of load, rate and indenter angle on the transformation behavior, Acta Mater. 53 (2005) 1759-1770. doi:10.1016/j.actamat.2004.12.025.

[10] Y.-H. Lin, T.-C. Chen, P.-F. Yang, S.-R. Jian, Y.-S. Lai, Atomic-level simulations of nanoindentation-induced phase transformation in mono-crystalline silicon, Appl. Surf. Sci. 254 (2007) 1415-1422. doi:10.1016/j.apsusc.2007.06.071.

[11] K. Mizushima, S. Yip, E. Kaxiras, Ideal crystal stability and pressure-induced phase transition in silicon, Phys. Rev. B. 50 (1994) 14952-14959. doi:10.1103/PhysRevB.50.14952.

[12] Y.B. Gerbig, C.A. Michaels, R.F. Cook, In situ observation of the spatial distribution of crystalline phases during pressure-induced transformations of indented silicon thin films, J. 
Mater. Res. 30 (2015) 390-406.

http://journals.cambridge.org/abstract_S0884291414003161 (accessed May 19, 2015).

[13] D. Clarke, M. Kroll, P. Kirchner, R. Cook, B. Hockey, Amorphization and Conductivity of Silicon and Germanium Induced by Indentation, Phys. Rev. Lett. 60 (1988) 2156-2159. doi:10.1103/PhysRevLett.60.2156.

[14] Y.. Wu, X.. Yang, Y.. Xu, Cross-sectional electron microscopy observation on the amorphized indentation region in [001] single-crystal silicon, Acta Mater. 47 (1999) 2431-2436. doi:10.1016/S1359-6454(99)00091-9.

[15] S. Goel, X. Luo, A. Agrawal, R.L. Reuben, Diamond machining of silicon: A review of advances in molecular dynamics simulation, Int. J. Mach. Tools Manuf. 88 (2015) 131-164. doi:10.1016/j.ijmachtools.2014.09.013.

[16] M. Gamero-Castaño, A. Torrents, L. Valdevit, J.-G. Zheng, Pressure-Induced Amorphization in Silicon Caused by the Impact of Electrosprayed Nanodroplets, Phys. Rev. Lett. 105 (2010) 145701. doi:10.1103/PhysRevLett.105.145701.

[17] M. Gamero-Castaño, A. Torrents, R. Borrajo-Pelaez, J.-G. Zheng, Amorphization of hard crystalline materials by electrosprayed nanodroplet impact, J. Appl. Phys. 116 (2014) 174309. doi:10.1063/1.4901287.

[18] F. Saiz, R. Borrajo-Pelaez, M. Gamero-Castaño, The influence of the projectile's velocity and diameter on the amorphization of silicon by electrosprayed nanodroplets, J. Appl. Phys. 114 (2013) 034304. doi:10.1063/1.4813416.

[19] S.K. Deb, M. Wilding, M. Somayazulu, P.F. McMillan, Pressure-induced amorphization and an amorphous-amorphous transition in densified porous silicon., Nature. 414 (2001) 528-30. doi:10.1038/35107036.

[20] V. Levitas, Crystal-Amorphous and Crystal-Crystal Phase Transformations via Virtual Melting, Phys. Rev. Lett. 95 (2005) 075701. doi:10.1103/PhysRevLett.95.075701.

[21] J. K. Lee, on cyclical phase transformations in driven alloy system, Metall. Mater. Trans. A. 39 (2007) 964-975.

[22] W.C. Johnson, J.K. Lee, G.J. Shiflet, Thermodynamic treatment of cyclic amorphization during ball milling, Acta Mater. 54 (2006) 5123-5133. doi:10.1016/j.actamat.2006.05.052. 
[23] L. Lei, T. Carvajal, K. Marisol, defect induced solid state amorphization of molecular crystals, J. Appl. Phys. 111 (2012) 073505.

[24] M.J. Demkowicz, A.S. Argon, Liquidlike atomic environments act as plasticity carriers in amorphous silicon, Phys. Rev. B. 72 (2005) 245205. doi:10.1103/PhysRevB.72.245205.

[25] V.I. Levitas, High-pressure mechanochemistry: Conceptual multiscale theory and interpretation of experiments, Phys. Rev. B - Condens. Matter Mater. Phys. 70 (2004) 1-24. doi:10.1103/PhysRevB.70.184118.

[26] A. Loveridge-Smith, A. Allen, J. Belak, T. Boehly, A. Hauer, B. Holian, et al., Anomalous Elastic Response of Silicon to Uniaxial Shock Compression on Nanosecond Time Scales, Phys. Rev. Lett. 86 (2001) 2349-2352. doi:10.1103/PhysRevLett.86.2349.

[27] R.F. Smith, C. A. Bolme, D.J. Erskine, P.M. Celliers, S. Ali, J.H. Eggert, et al., Heterogeneous flow and brittle failure in shock-compressed silicon, J. Appl. Phys. 114 (2013) 133504. doi:10.1063/1.4820927.

[28] H. Kishimura, H. Matsumoto, N.N. Thadhani, Effect of shock compression on single crystalline silicon, J. Phys. Conf. Ser. 215 (2010) 012145.

doi:10.1088/1742-6596/215/1/012145.

[29] G. Mogni, A. Higginbotham, K. Gaál-Nagy, N. Park, J.S. Wark, Molecular dynamics simulations of shock-compressed single-crystal silicon, Phys. Rev. B. 89 (2014) 064104. doi:10.1103/PhysRevB.89.064104.

[30] S. Zhao, B. Kad, E. Hahn, B.A. Remington, C.E. Wehrenburg, C.M. Huntington, et al., No Title, Submitted. (2015).

[31] D.J. Gardiner, P.R. Graves, eds., Practical Raman Spectroscopy, Springer Berlin Heidelberg, Berlin, Heidelberg, 1989. doi:10.1007/978-3-642-74040-4.

[32] S. Plimpton, Fast Parallel Algorithms for Short-Range Molecular Dynamics, J. Comput. Phys. 117 (1995) 1-19. doi:10.1006/jcph.1995.1039.

[33] T. Kumagai, S. Izumi, S. Hara, S. Sakai, Development of bond-order potentials that can reproduce the elastic constants and melting point of silicon for classical molecular dynamics simulation, Comput. Mater. Sci. 39 (2007) 457-464.

doi:10.1016/j.commatsci.2006.07.013. 
[34] D. Tramontina, P. Erhart, T. Germann, J. Hawreliak, A. Higginbotham, N. Park, et al., Molecular dynamics simulations of shock-induced plasticity in tantalum, High Energy Density Phys. 10 (2014) 9-15. doi:10.1016/j.hedp.2013.10.007.

[35] A. Stukowski, Visualization and analysis of atomistic simulation data with OVITO-the Open Visualization Tool, Model. Simul. Mater. Sci. Eng. 18 (2010) 015012. doi:10.1088/0965-0393/18/1/015012.

[36] J.M.D. Lane, A.P. Thompson, T.J. Vogler, Enhanced densification under shock compression in porous silicon, Phys. Rev. B. 90 (2014) 134311. doi:10.1103/PhysRevB.90.134311.

[37] A. T. Voutsas, M.K. Hatalis, J. Boyce, A. Chiang, Raman spectroscopy of amorphous and microcrystalline silicon films deposited by low-pressure chemical vapor deposition, J. Appl. Phys. 78 (1995) 6999. doi:10.1063/1.360468.

[38] G. Gouadec, P. Colomban, Raman Spectroscopy of Nanomaterials: How Spectra Relate to Disorder, Particle size and Mechanical Properties, in: Prog. Cryst. Growth Charact. Mater., 2007: pp. 1-56. doi:<10.1016/j.pcrysgrow.2007.01.001>.

[39] C. Fontaine, On the atomic structure of the $\Sigma=3,\{112\}$ twin in silicon, Appl. Phys. Lett. 40 (1982) 153. doi:10.1063/1.93019.

[40] S.M. Sharma, S.K. Sikka, Pressure induced amorphization of materials, Prog. Mater. Sci. 40 (1996) 1-77. doi:10.1016/0079-6425(95)00006-2.

[41] O. Mishima, L.D. Calvert, E. Whalley, "Melting ice" I at $77 \mathrm{~K}$ and $10 \mathrm{kbar}$ : a new method of making amorphous solids, Nature. 310 (1984) 393-395. doi:10.1038/310393a0.

[42] R.J. Hemley, A.P. Jephcoat, H.K. Mao, L.C. Ming, M.H. Manghnani, Pressure-induced amorphization of crystalline silica, Nature. 334 (1988) 52-54. doi:10.1038/334052a0.

[43] M. Chen, J.W. McCauley, K.J. Hemker, Shock-induced localized amorphization in boron carbide., Science (80-. ). 299 (2003) 1563-1566. doi:10.1126/science.1080819.

[44] X. Lü, Q. Hu, W. Yang, L. Bai, H. Sheng, L. Wang, et al., Pressure-induced amorphization in single-crystal Ta2O5 nanowires: a kinetic mechanism and improved electrical conductivity., J. Am. Chem. Soc. 135 (2013) 13947-53. doi:10.1021/ja407108u. 
[45] D. Machon, F. Meersman, M.C. Wilding, M. Wilson, P.F. McMillan, Pressure-induced amorphization and polyamorphism: Inorganic and biochemical systems, Prog. Mater. Sci. 61 (2014) 216-282. doi:10.1016/j.pmatsci.2013.12.002.

[46] R. Lagneborg, The martensite transformation in $18 \%$ Cr- $8 \%$ Ni steels, Acta Metall. 12 (1964) 823-843.

[47] M.W. Chase, J.L. Curnutt, H. Prophet, R.A. McDonald, A.N. Syverud, JANAF thermochemical tables, J. Phys. Chem. Ref. Data. 4 (1975) 1-176.

[48] P. Bridgman, Effects of High Shearing Stress Combined with High Hydrostatic Pressure, Phys. Rev. 48 (1935) 825-847. doi:10.1103/PhysRev.48.825.

[49] G. Duvall, R Graham, Phase transitions under shock-wave loading, Rev. Mod. Phys. 49 (1977) 523-579.

[50] H.C. Chen, J.C. Lasalvia, V.F. Nesterenko, M.A. Meyers, Shear localization and chemical reaction in high-strain, high strain-rate deformation of Ti-Si powder mixtures, Acta Mater. 46 (1998) 3033-3046.

[51] J.R. Patel, M. Cohen, criterion for the action of applied stress in the martensitic transformation, Acta Metall. 1 (1953) 531-538.

[52] N.N. Thadhanit, M.A. Meyers, Kinetics of martensitic transformation induced by a tensile stress pulse, Acta Metall. 34 (1986) 1625-1641.

[53] M.A. Meyers, Dynamic behavior of materials, John Wiley \& Sons, 1994.

[54] D.A. Porter, K.E. Easterling, M.Y. Sherif, Phase transformations in metals and alloys, 3rd ed., Taylor \& Francis, 2009.

[55] S. Amelinckx, Dislocations in Particular Structures, in: F.R.N. Nabarro (Ed.), Dislocations in Solids, North-Holland, Amsterdam, 1982: pp. 67-460.

[56] D. Roundy, C.R. Krenn, M.L. Cohen, J.W. Morris, Ideal Shear Strengths of fcc Aluminum and Copper, Phys. Rev. Lett. 82 (1999) 2713-2716. doi:10.1103/PhysRevLett.82.2713.

[57] S. Kibey, J.B. Liu, D.D. Johnson, H. Sehitoglu, Predicting twinning stress in fcc metals: Linking twin-energy pathways to twin nucleation, Acta Mater. 55 (2007) 6843-6851. doi:10.1016/j.actamat.2007.08.042. 
[58] J.P.Hirth, Jens Lothe, Theory of dislocations, 2nd ed., John Wiley \& Sons, New York, 1982.

[59] E. Aerts, P. Dellavignette, R. Siems, S. Amelinckx, Stacking fault energy in silicon, J. Appl. Phys. 33 (1962) 3078-3080.

[60] M. A. Hopcroft, W.D. Nix, T.W. Kenny, What is the Young's Modulus of Silicon?, J. Microelectromechanical Syst. 19 (2010) 229-238. doi:10.1109/JMEMS.2009.2039697.

[61] Q.S. Mei, K. Lu, Melting and superheating of crystalline solids: From bulk to nanocrystals, Prog. Mater. Sci. 52 (2007) 1175-1262. doi:10.1016/j.pmatsci.2007.01.001.

[62] M.A. Meyers, H. Jarmakani, E.M. Bringa, B.A. Remington, Dislocations in Shock Compression and Release, in: Dislocations in Solids, Chapter 89, 2009: pp. 91-197.

[63] V.I. Levitas, M. Javanbakht, Phase transformations in nanograin materials under high pressure and plastic shear: nanoscale mechanisms., Nanoscale. 6 (2014) 162-6. doi:10.1039/c3nr05044k.

[64] H. Gleiter, B. Chalmers, High-angle grain boundaries, Pergamon Press, new york, 1972. http://books.google.com/books/about/High_angle_grain_boundaries.html?id=PUhSAAA AMAAJ\&pgis=1 (accessed November 10, 2014).

[65] R.D. Doherty, D.A. Hughes, F.J. Humphreys, J.J. Jonas, D.J. Jensen, M.E. Kassner, et al., Current issues in recrystallization: a review, Mater. Sci. Eng. A. 238 (1997) 219-274. doi:10.1016/S0921-5093(97)00424-3.

[66] A. Otsuki, Energies of (001) twist grain boundaries in silicon, Acta Mater. 49 (2001) 1737-1745. doi:10.1016/S1359-6454(01)00090-8.

[67] S. Costantini, P. Alippi, L. Colombo, F. Cleri, Triple junctions and elastic stability of polycrystalline silicon, Phys. Rev. B. 63 (2000) 045302. doi:10.1103/PhysRevB.63.045302.

[68] T. Cramer, a. Wanner, P. Gumbsch, Energy Dissipation and Path Instabilities in Dynamic Fracture of Silicon Single Crystals, Phys. Rev. Lett. 85 (2000) 788-791. doi:10.1103/PhysRevLett.85.788.

[69] Y. Mishin, M. Asta, J. Li, Atomistic modeling of interfaces and their impact on microstructure and properties, Acta Mater. 58 (2010) 1117-1151. doi:10.1016/j.actamat.2009.10.049. 
[70] G. Gottstein, L.S. Shvindlerman, Triple junction drag and grain growth in 2D polycrystals, Acta Mater. 50 (2002) 703-713. doi:10.1016/S1359-6454(01)00391-3.

[71] G. Gottstein, L.S. Shvindlerman, Grain Boundary Migration in Metals: Thermodynamics, Kinetics, Applications, CRC Press, 1999.

http://books.google.com/books?hl=en\&lr=\&id=pKaFe6njzdUC\&pgis=1 (accessed November 10, 2014).

[72] H. Hu, B.B. Rath, On the time exponent in isothermal grain growth, Metall. Trans. 1 (n.d.) 3181-3184. doi:10.1007/BF03038435.

[73] C.H. Lu, B.A. Remington, B.R. Maddox, B. Kad, H.S. Park, M. Kawasaki, et al., Laser compression of nanocrystalline tantalum, Acta Mater. 61 (2013) 7767-7780. doi:10.1016/j.actamat.2013.09.016.

[74] G. Gottstein, L.S. Shvindlerman, B. Zhao, Thermodynamics and kinetics of grain boundary triple junctions in metals: Recent developments, Scr. Mater. 62 (2010) 914-917. doi:10.1016/j.scriptamat.2010.03.017.

[75] B. Zhao, G. Gottstein, L.S. Shvindlerman, Triple junction effects in solids, Acta Mater. 59 (2011) 3510-3518. doi:10.1016/j.actamat.2011.02.024.

[76] M. Asta, C. Beckermann, A. Karma, W. Kurz, R. Napolitano, M. Plapp, et al., Solidification microstructures and solid-state parallels: Recent developments, future directions, Acta Mater. 57 (2009) 941-971. doi:10.1016/j.actamat.2008.10.020.

[77] F. Ye, K. Lu, Crystallization kinetics of amorphous solids under pressure, Phys. Rev. B. 60 (1999) 7018-7024. doi:10.1103/PhysRevB.60.7018.

[78] F.H. Streitz, J.N. Glosli, M. V. Patel, Beyond finite-size scaling in solidification simulations, Phys. Rev. Lett. 96 (2006). doi:10.1103/PhysRevLett.96.225701.

\section{Table}

Table 1. Calculated values of shock pressure, hydrostatic pressure and maximum shear stress.

\begin{tabular}{cllll}
\hline $\mathrm{E}_{\text {laser }}$ & $P_{\mathrm{zz}}$ & $\mathrm{P}$ & $\tau_{\max }$ & $\frac{\tau_{\max }}{P}=\frac{3\left(C_{11}-C_{12}\right)}{2\left(C_{11}+2 C_{12}\right)}$ \\
{$[J]$} & {$[\mathrm{GPa}]$} & {$[\mathrm{GPa}]$} & {$[\mathrm{GPa}]$} & $P$ \\
\hline
\end{tabular}




\begin{tabular}{ccccc}
\hline 18.2 & 4.1 & 2.4 & 1.2 & 0.5 \\
50.4 & 11 & 7.2 & 2.9 & 0.4 \\
106.8 & 22.3 & 14.3 & 4.3 & 0.3 \\
\hline
\end{tabular}




\section{Figure Captions}

Fig. 1. Schematic drawing of the laser shock compression experiment set-up (upper panel); details of the target package (bottom-left panel); peak pressure as function of laser energy (bottom-right panel).

Fig. 2. Raman spectra of the laser shock-recovered silicon targets.

Fig. 3. TEM images of the complex amorphous pattern: (a) broad region of the amorphous material on the top surface, marked by A, below which multiple amorphous bands penetrate into the crystal ; (b) pattern of of the amorphous bands (B) decreasing in thickness with depth; (c) termination (D), bifurcations and feathering, marked by C, usually observed along with the primary bands and different variants of the bands eventually intersect; (d) the intersection leads to the formation of a jog/kink feature and is indicated by $\mathrm{E}$.

Fig.4 High resolution TEM micrographs showing the onset of amorphous bands: (a) a single amorphous band surrounded by stacking faults; (b) inverse FFT image of the boxed region in (a), showing clearly the zigzag feature of multiple SFs; (c) nucleation of the amorphization at the SFs intersections; (d) inverse FFT image of the boxed region in (c) shows that the intersected region become less order.

Fig. 5. (a) (001) Stereographic projection with maximum shear cone indicated by red dashed circle. (b) Detail of stereographic projection. TEM samples always show a foil normal of $\{110\}$ and the corresponding slip and twinning planes are marked by green ( $\{111\}$ planes) and blue ( $\{112\})$, respectively.

Fig. 6. High resolution TEM image of an amorphous band initiated along $\{111\}$ slip planes $\left(35.3^{0}\right.$ with shock direction) and spreading towards the direction of the maximum shear $\left(45^{0}\right.$ with shock direction). Adjoining stacking faults marked SF.

Fig. 7. Molecular dynamics snapshot at $15 \mathrm{ps}$ of a [001] silicon crystal shocked to $14.5 \mathrm{GPa}$ From left to right: an orthogonal view of defective atoms, [110] projection of defective atoms, and an atom slice colored by local atomic coordination. All further figures are colored consistently.

Fig. 8. Stepped stacking fault band with amorphous interior. $\{111\}$ stacking faults make a projection of $54^{\circ}$ and bands deviate by stepping towards maximum shear at $45^{\circ}$.

Fig. 9 Schematic illustration of amorphous band formation through the passage of one set of stacking faults. 
Fig. 10 Schematic illustration of the amorphous phase nucleated from stacking-fault intersections.

Fig. 11. Molecular dynamics of a [001] silicon crystal shocked to $14.5 \mathrm{GPa}$ showing time evolution. Formation and expansion of fully transformed region initiating at surface followed by pressure-shear driven stacking-fault bands which amorphize on thickening. Notice intersection of stacking-fault bands leading to amorphization.

Fig. 12 (a) Gibbs free energy of a-Si and c-Si as function of T, the values of a-Si is assumed to be identical with liquid silicon; the difference between a-Si and c-Si is denoted as $\Delta G_{c-a}$, the energy barrier for the c-a transformation. (b) The influence of pressure and shear on the energy barrier. (c) Calculated c-a transformation temperature and shock temperature as function of shock pressure.

Fig. 13. Influence of defect density (in $\mathrm{cm}^{-2}$ ) on the Gibbs free energy for amorphization nucleation at a constant pressure $\left(P_{\text {shock }} \sim 11 \mathrm{GPa}, \Delta \mathrm{G}_{\mathrm{c}-\mathrm{a}} \sim 5 \mathrm{~kJ} / \mathrm{mol}\right.$ at room temperature $)$.

Fig. 14. Calculated dislocation density $\left(\rho_{d}\right)$ as a function of pressure $P$ assuming homogeneous nucleation of loops behind front [56]. Hugoniot relationship of the modified Tersoff potential used to obtain pressure vs. volume. Experimental laser experiments marked on plot by their energy levels. The shaded cyan area represents the range of the predicted Hugoniot elastic limit (HEL). The dotted lines denote the shock pressure threshold (and corresponding dislocation density at shock front) where amorphization is observed experimentally.

Fig. 15. TEM micrographs of the $150 \mathrm{~J}$-shocked silicon crystal showing crystallization of amorphous structure: a, top surface showing grain size of around one micrometer; $b, \sim 3 \mu \mathrm{m}$ below the surface showing a ultrafine grained structure; $\mathrm{c}, \sim 7 \mu \mathrm{m}$ below the surface showing even finer grain size; $\mathrm{d}, \sim 10 \mu \mathrm{m}$ below the surface showing a mixture of amorphous band and undeformed monocrystalline crystal.

Fig. 16. Grain size distribution of the $150 \mathrm{~J}$-shocked sample as function of depth below the shock surface. Four regions, namely coarse grained (CG-Si); ultrafine grained (UFG-Si); nanocrystalline (nc-Si); and mixture of a-Si and monocrystalline ( $\mathrm{m}-\mathrm{Si}$ ), can be classified depend on the grain size. Fig. 17. (a) HRTEM micrograph of a nucleus crystallized from amorphous silicon; (b) magnified view of the black boxed region in (a), showing the crystalline embryo (delineated by white dashed line) with clear twined structure. 


\section{Figures}

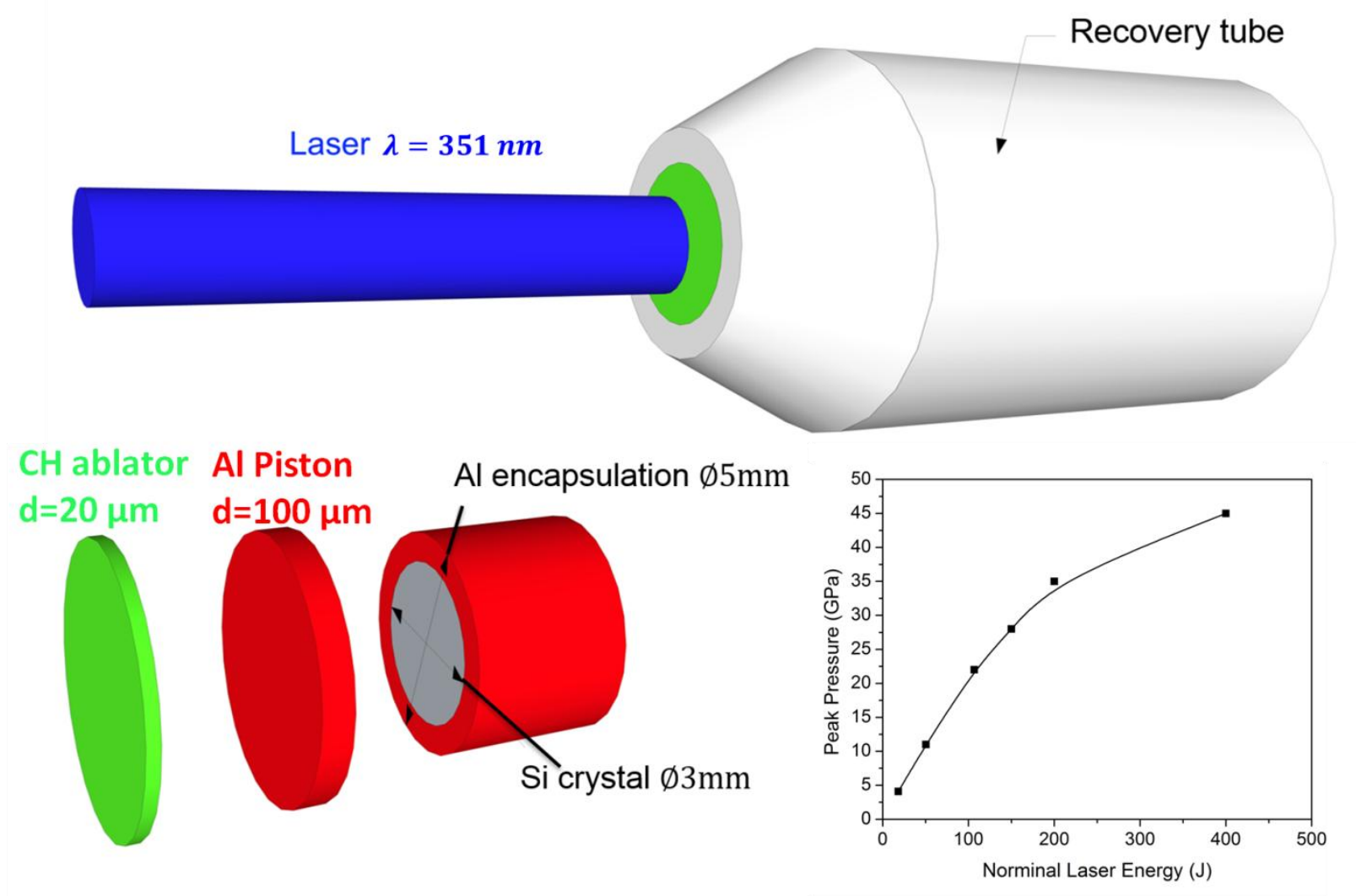

Fig. 1. Schematic drawing of the laser shock compression experiment set-up (upper panel); details of the target package (bottom-left panel); peak pressure as function of laser energy (bottom-right panel). 


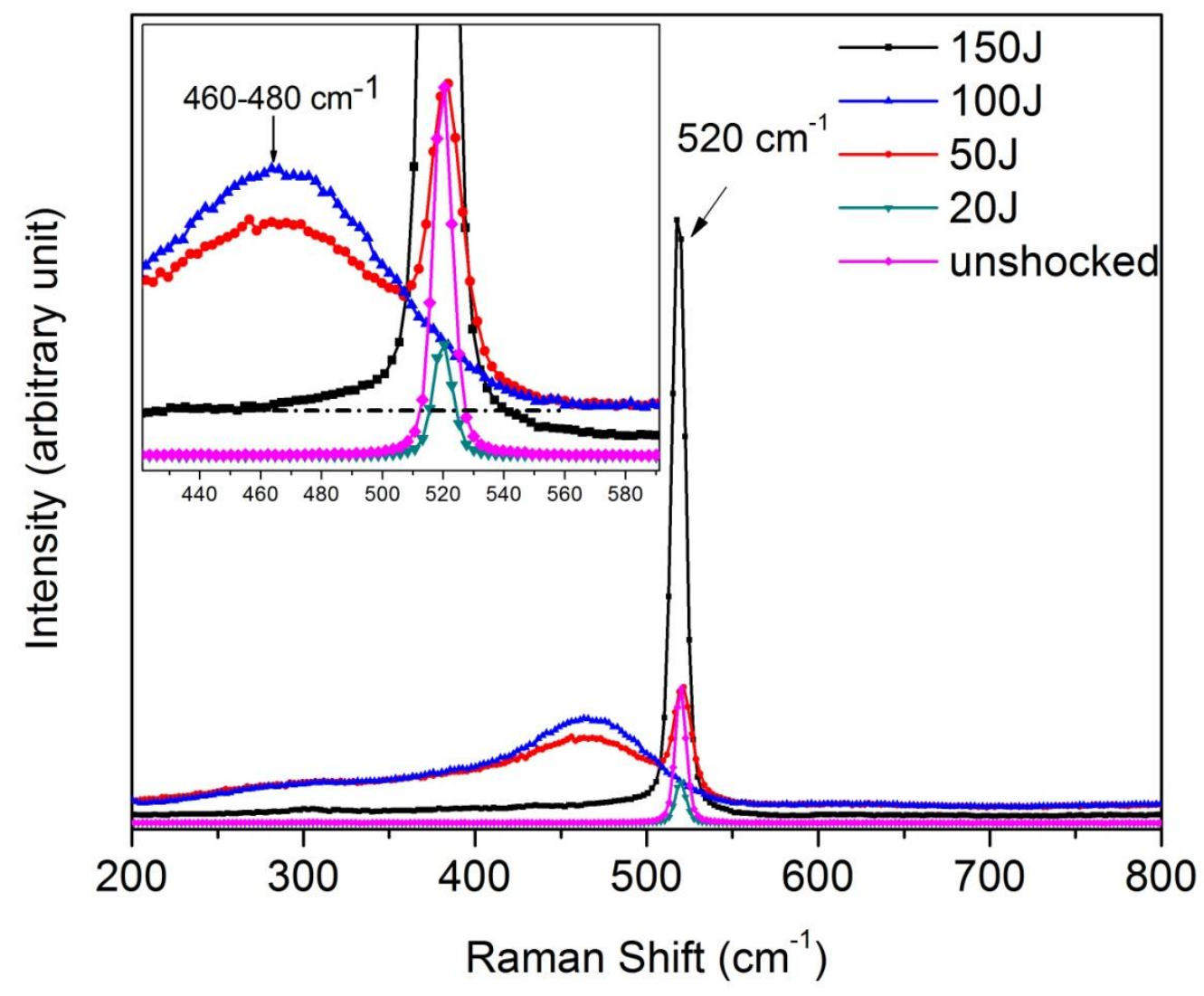

Fig. 2. Raman spectra of the laser shock-recovered silicon targets. 

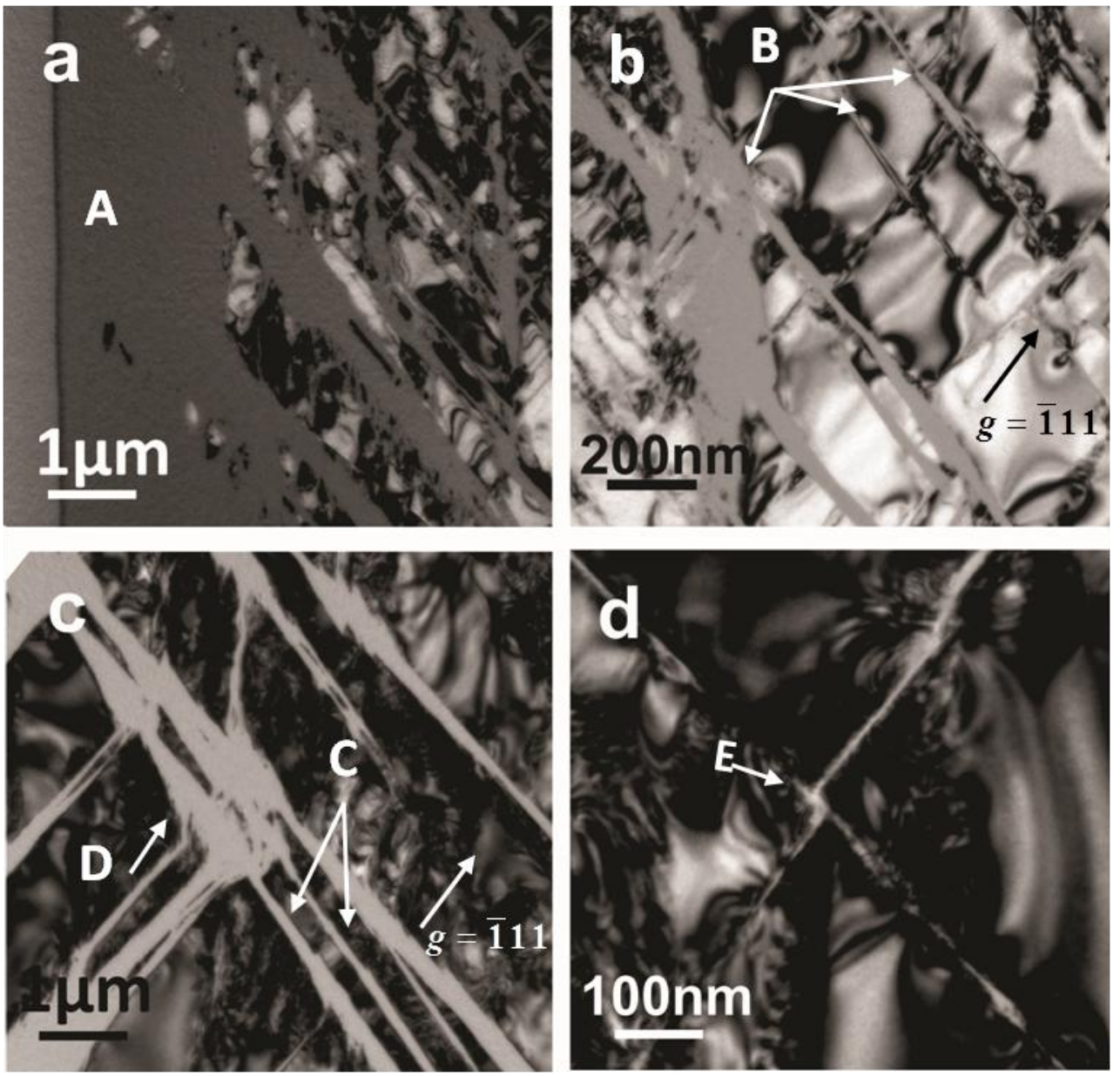

Fig. 3. TEM images of the complex amorphous pattern: (a) broad region of the amorphous material on the top surface, marked by A, below which multiple amorphous bands penetrate into the crystal ; (b) pattern of the amorphous bands (B) decreasing in thickness with depth; (c) termination (D), bifurcations and feathering, marked by $\mathrm{C}$, usually observed along with the primary bands and different variants of the bands eventually intersect; (d) the intersection leads to the formation of a jog/kink feature and is indicated by $\mathrm{E}$. 

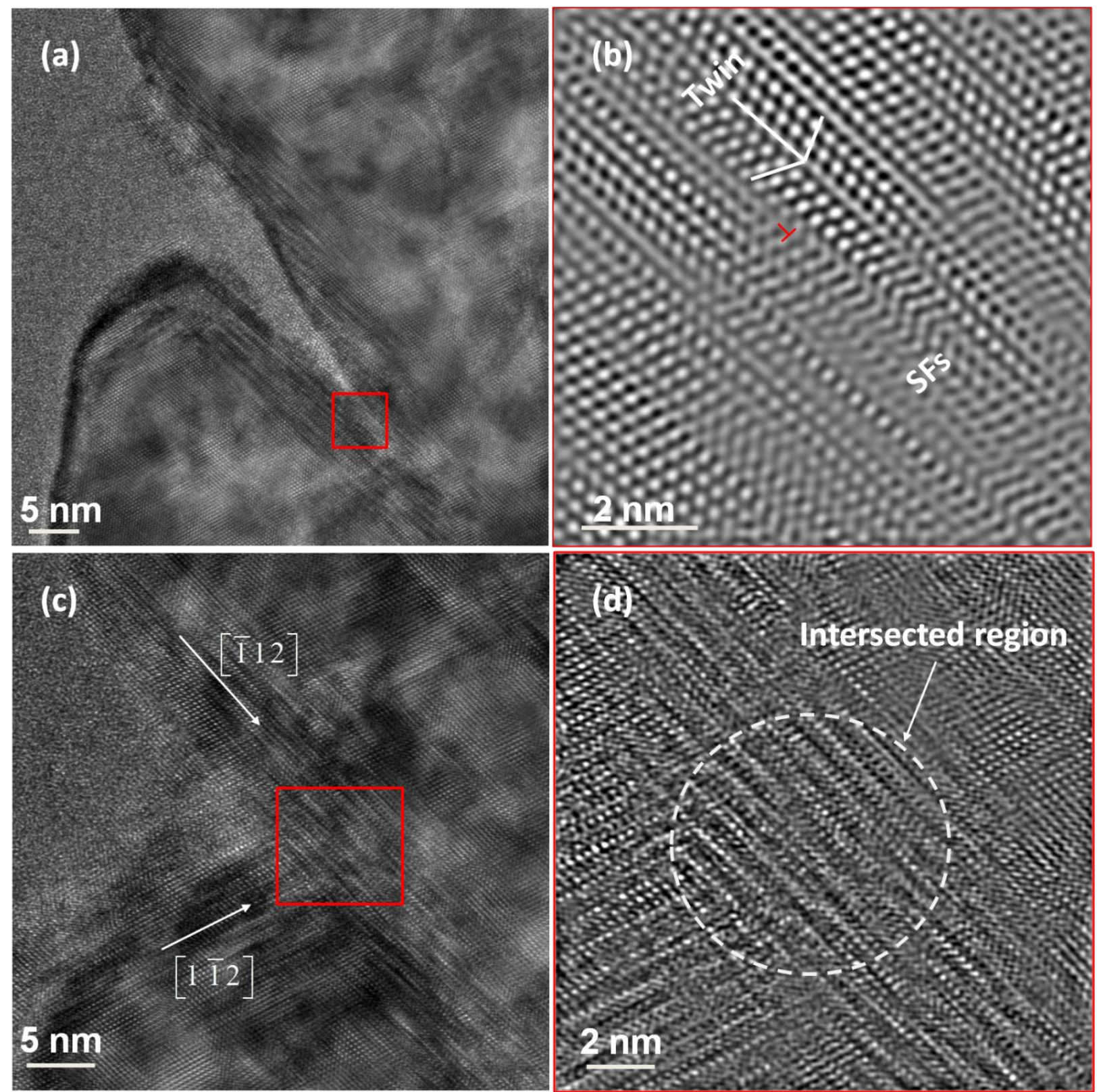

Fig.4. High resolution TEM micrographs showing the onset of amorphous bands: (a) a single amorphous band surrounded by stacking faults; (b) inverse FFT image of the boxed region in (a), showing clearly the zigzag feature of multiple SFs, twins and dislocation; (c) nucleation of the amorphization at the SFs intersections; (d) inverse FFT image of the boxed region in (c) shows that the intersected region become less order. 


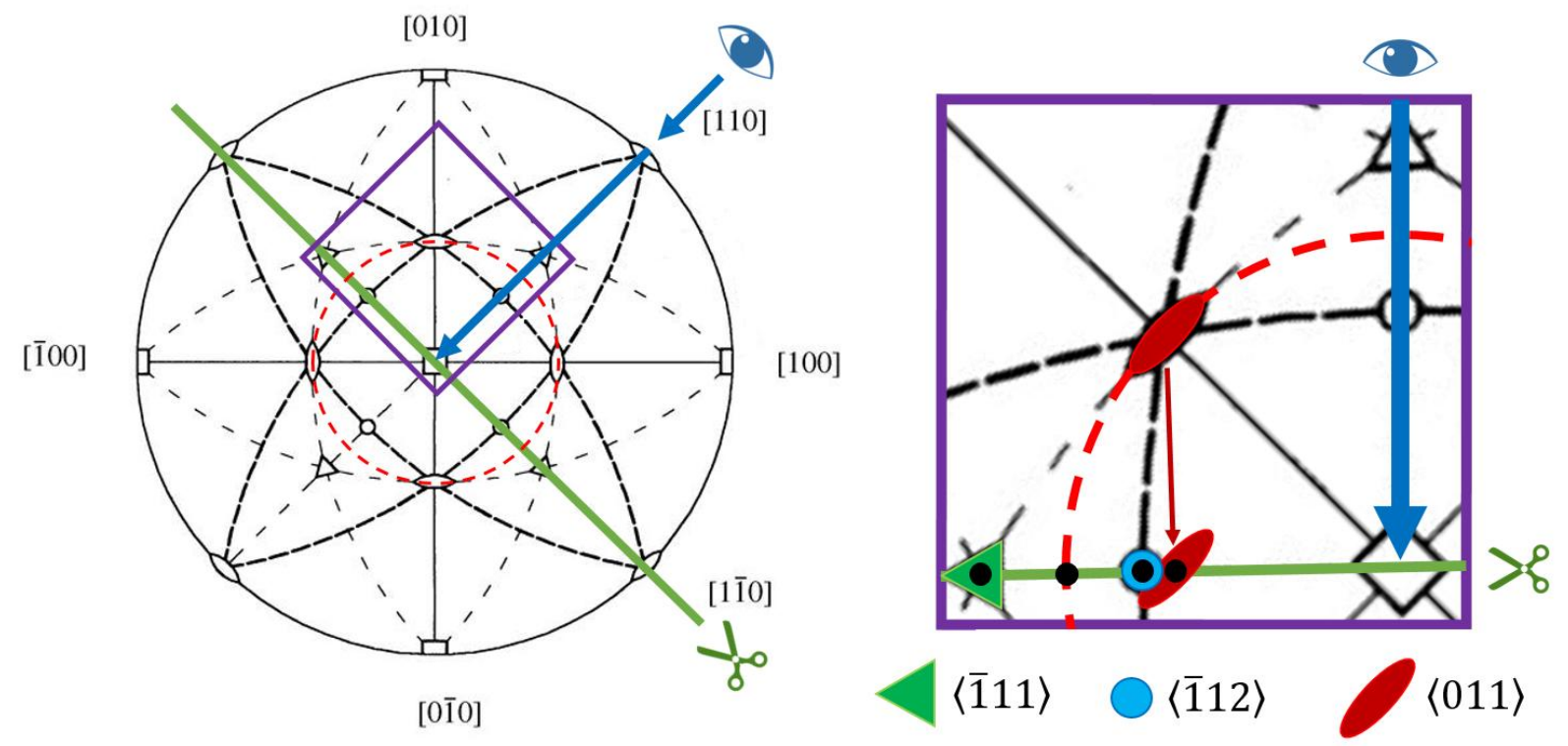

Fig. 5. (a) (001) Stereographic projection with maximum shear cone indicated by red dashed circle. (b) Detail of stereographic projection. TEM samples always show a foil normal of $\{110\}$ and the corresponding slip and twinning planes are marked by green (\{111\} planes) and blue $(\{112\})$, respectively. 


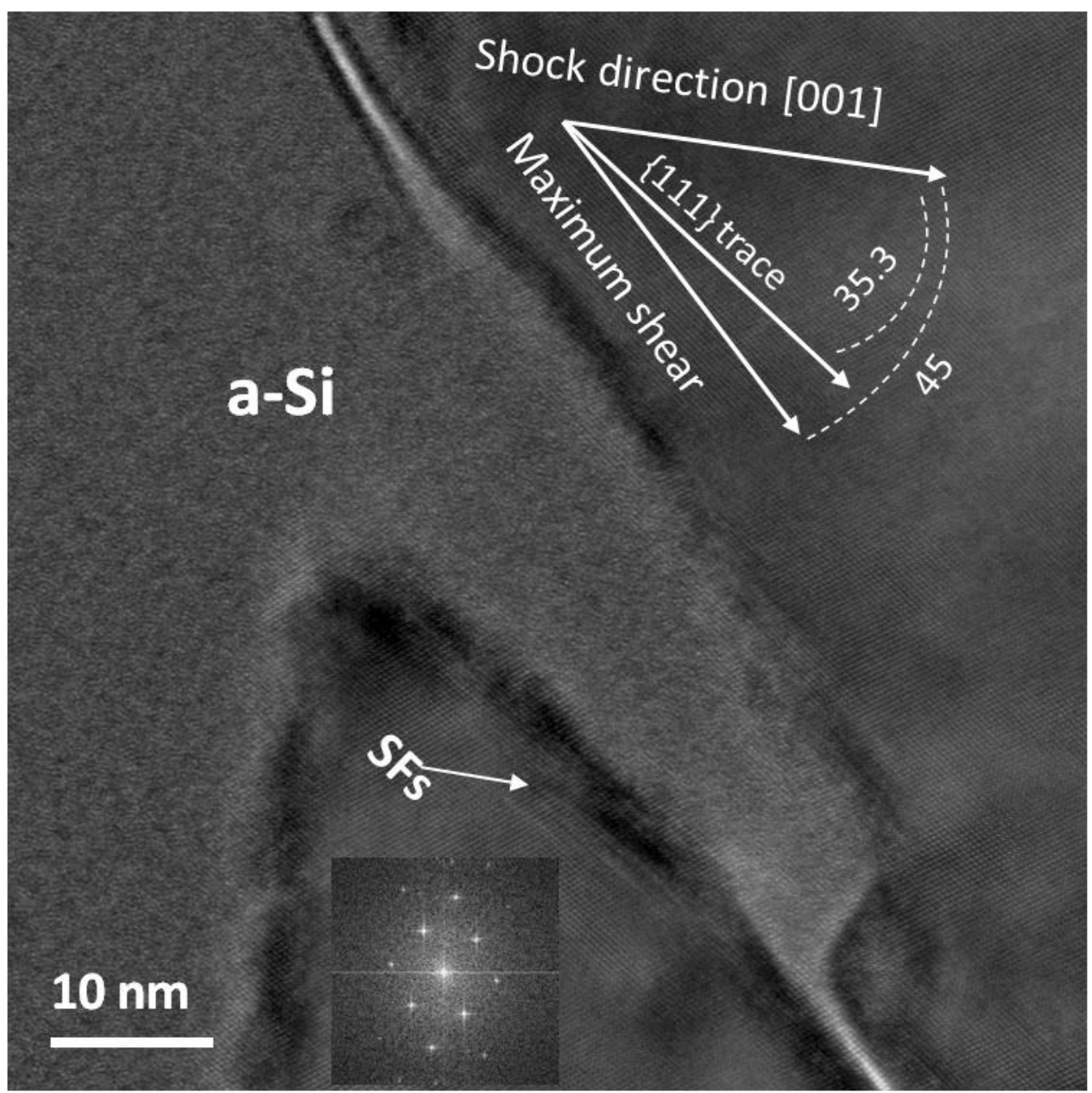

Fig. 6. High resolution TEM image of an amorphous band initiated along $\{111\}$ slip planes $\left(35.3^{0}\right.$ with shock direction) and spreading towards the direction of the maximum shear $\left(45^{0}\right.$ with shock direction). Adjoining stacking faults marked SF. 


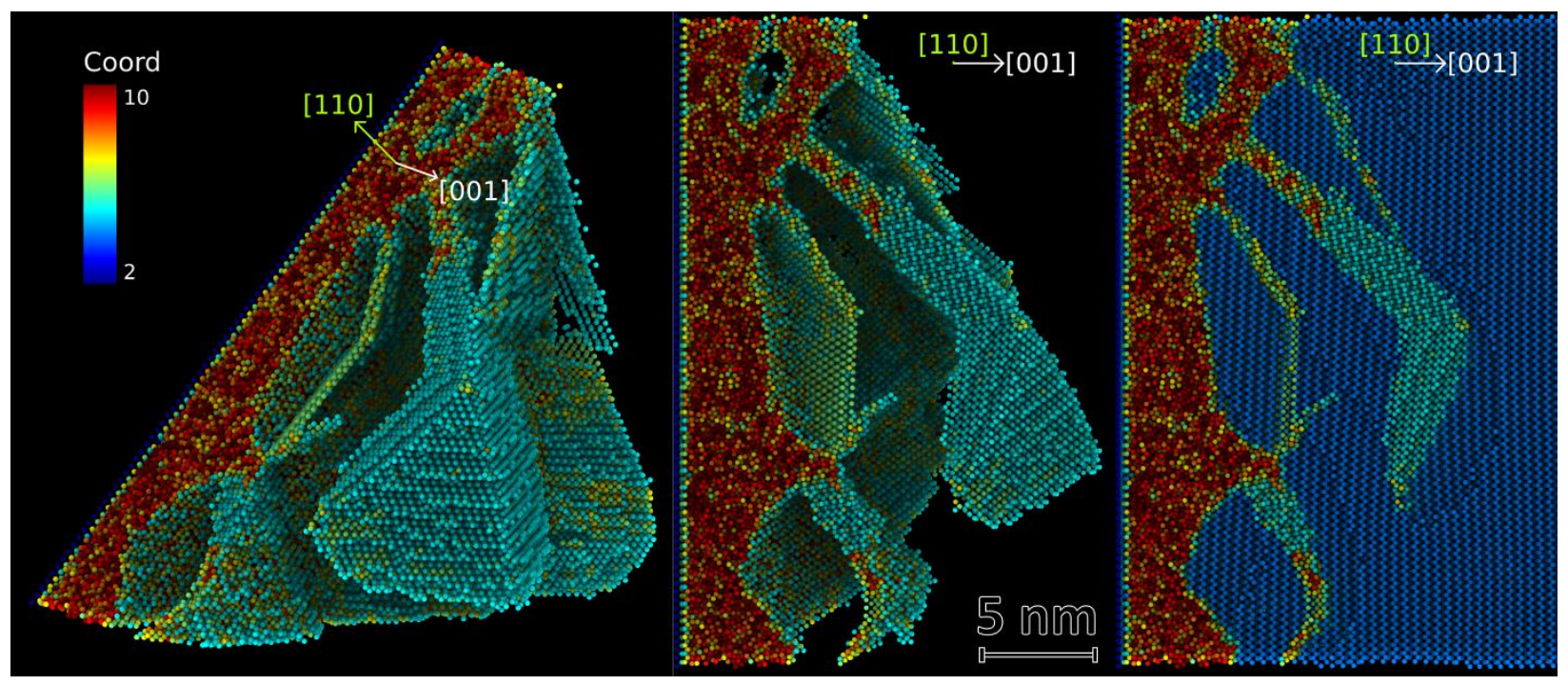

Fig. 7. Molecular dynamics snapshot at $15 \mathrm{ps}$ of a [001] silicon crystal shocked to $14.5 \mathrm{GPa}$. From left to right: an orthogonal view of defective atoms, [110] projection of defective atoms, and an atom slice colored by local atomic coordination. All further figures are colored consistently.

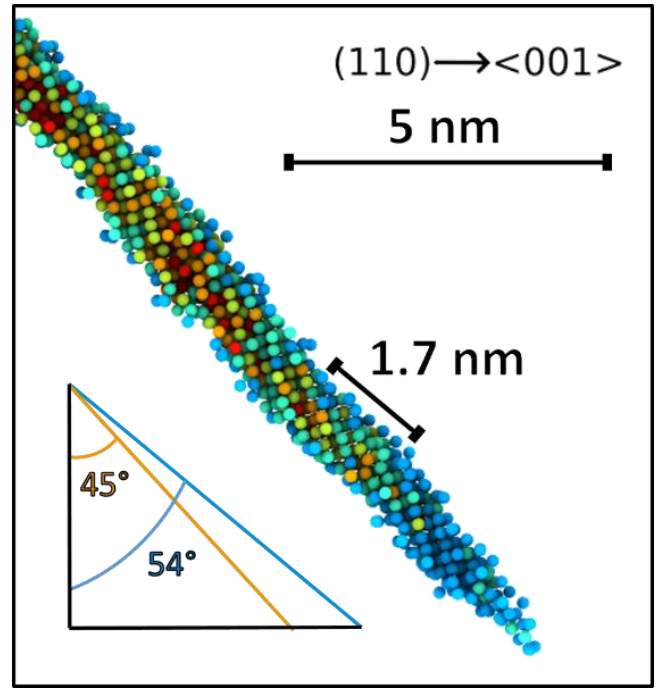

Fig. 8. Stepped stacking fault band with amorphous interior. $\{111\}$ stacking faults make a projection of $54^{\circ}$ and bands deviate by stepping towards maximum shear at $45^{\circ}$. 


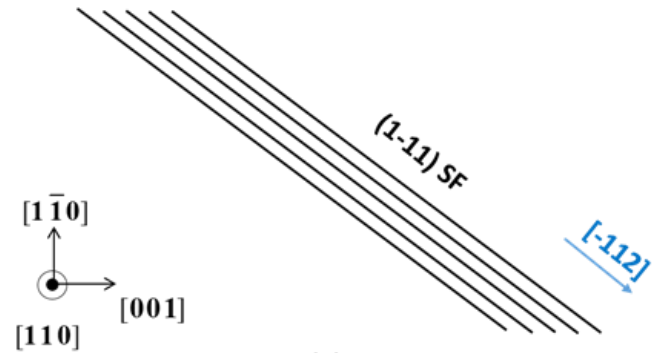

(a)

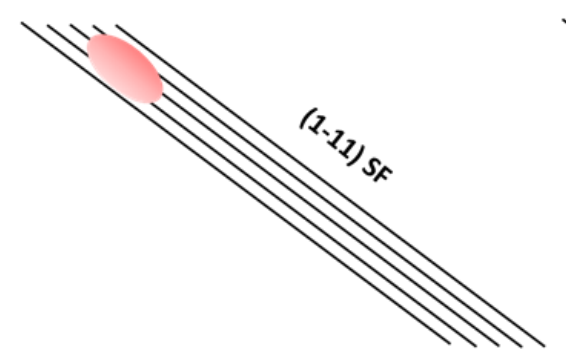

(b)

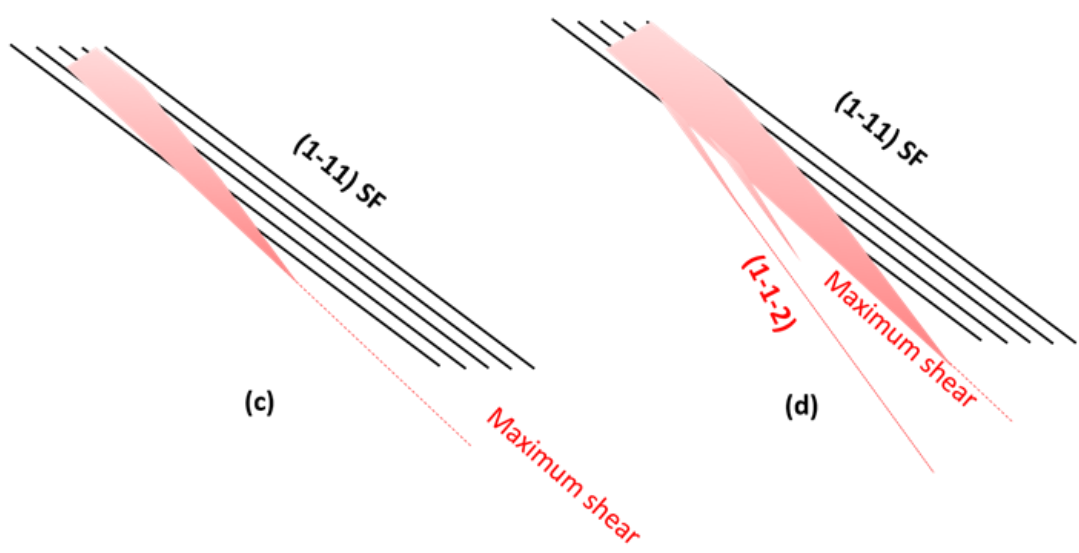

Fig. 9. Schematic illustration of amorphous band nucleation inside set of stacking faults and growth along maximum shear and (112)

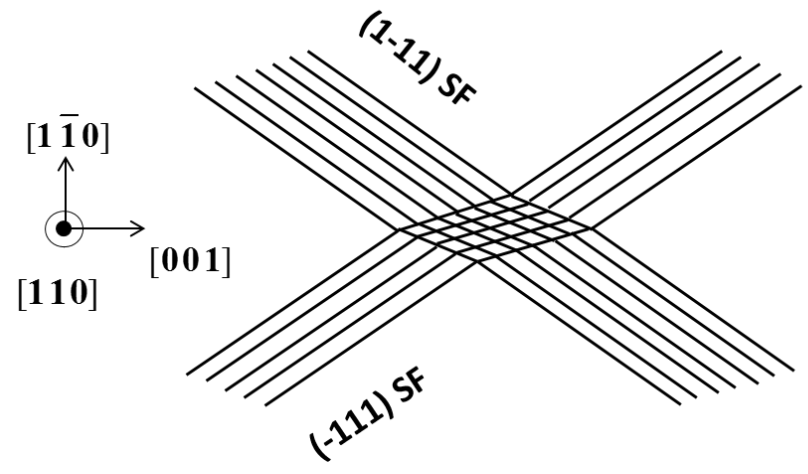

(a)

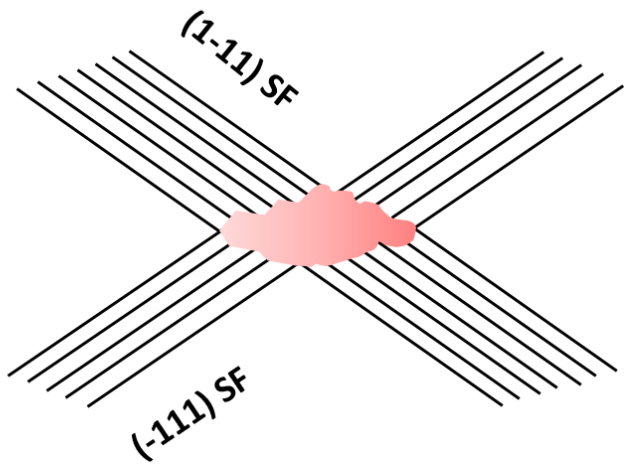

(b)

Fig. 10. Schematic illustration of the amorphous phase nucleated from stacking-fault intersections. 

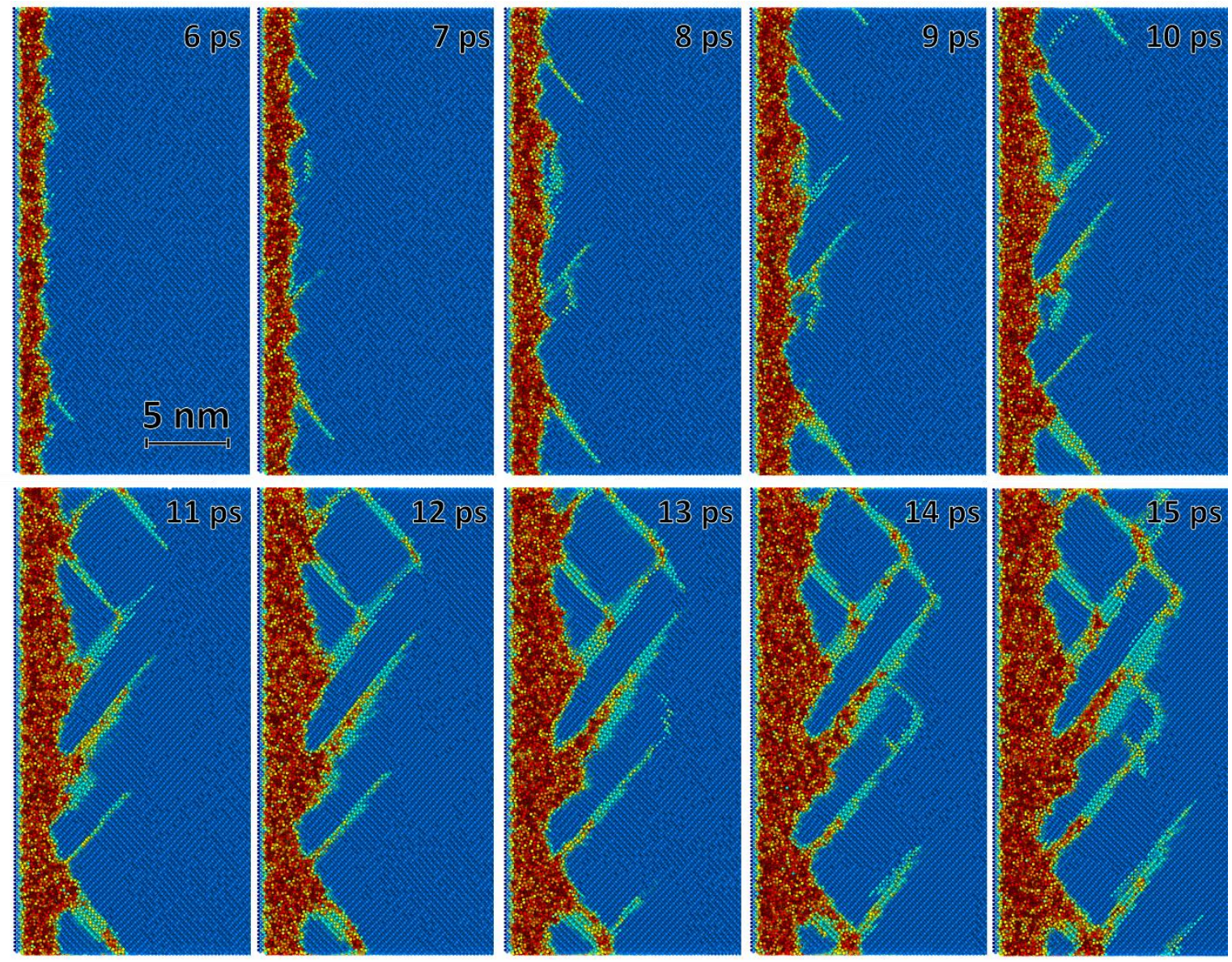

Fig. 11. Molecular dynamics of a [001] silicon crystal shocked to $14.5 \mathrm{GPa}$ showing time evolution. Formation and expansion of fully transformed region initiating at surface followed by pressure-shear driven stacking-fault bands which amorphize on thickening. Notice intersection of stacking-fault bands leading to amorphization. 

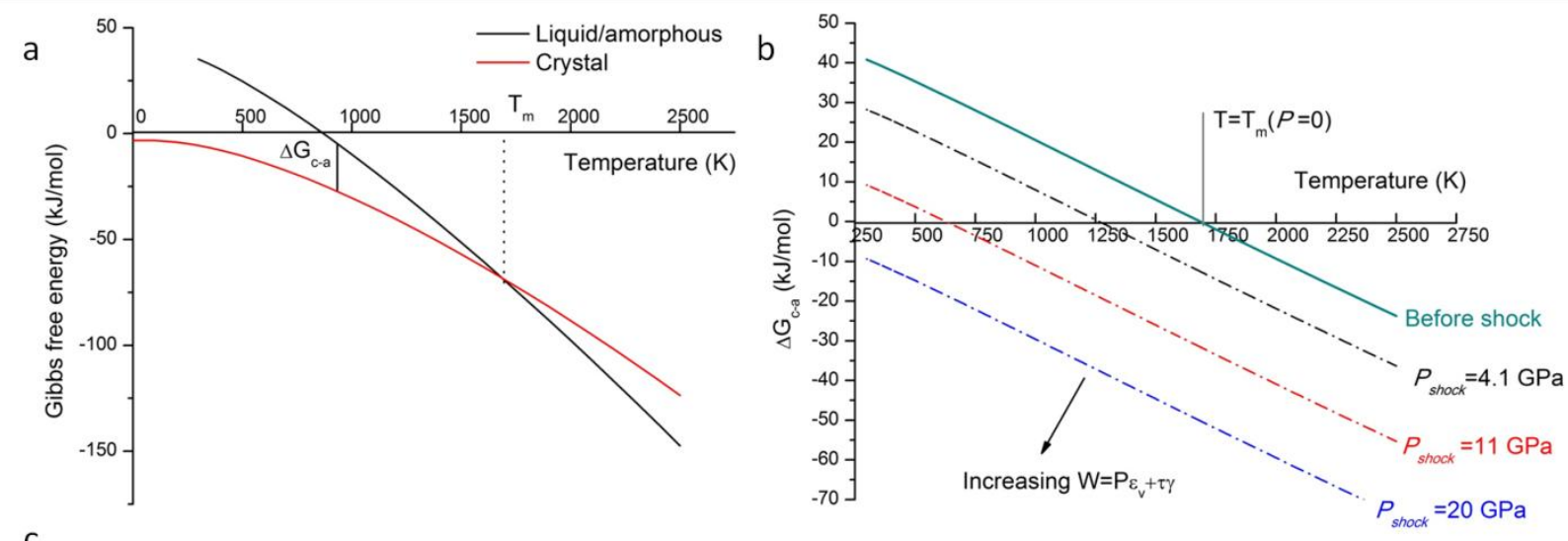

C

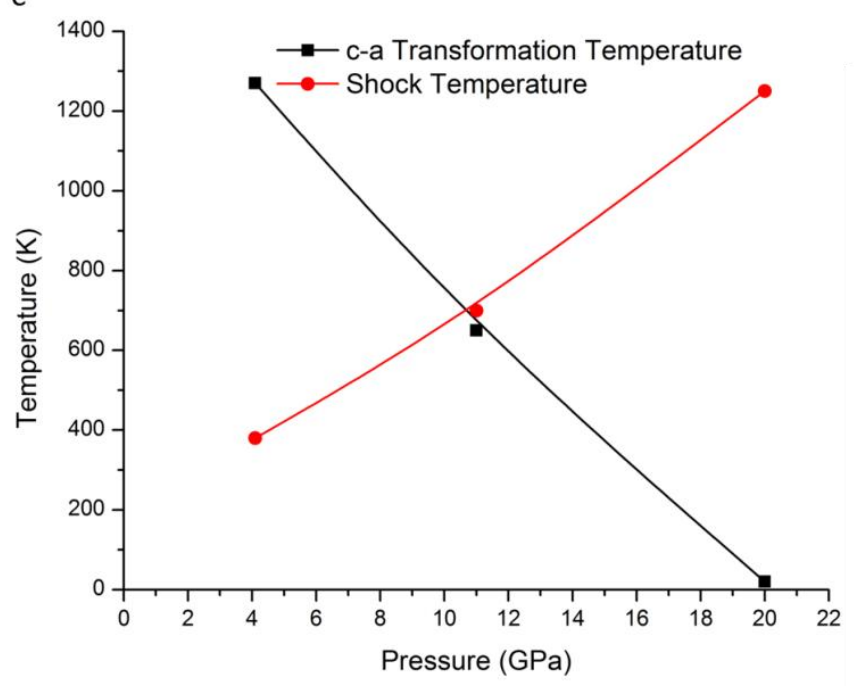

Fig. 12. (a) Gibbs free energy of a-Si and c-Si as function of T from [41], the values of a-Si is assumed to be identical with liquid silicon; the difference between a-Si and c-Si is denoted as $\Delta G_{c-a}$, the energy barrier for the c-a transformation. (b) The influence of pressure and shear on the energy barrier. (c) Calculated c-a transformation temperature and shock temperature as function of shock pressure. 


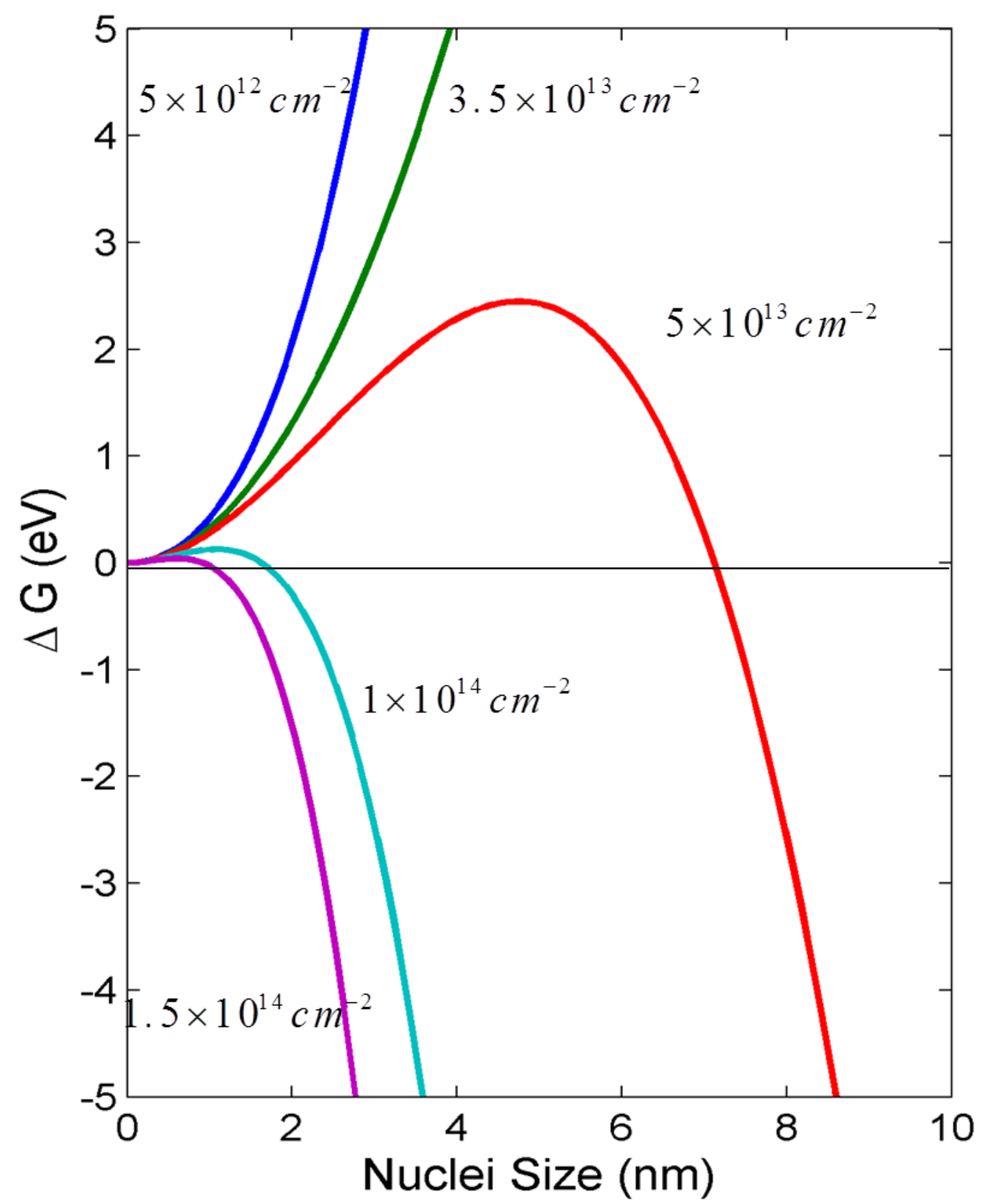

Fig. 13. Influence of defect density (in $\mathrm{cm}^{-2}$ ) on the Gibbs free energy for amorphization nucleation at a constant pressure ( $P_{\text {shock }} \sim 11 \mathrm{GPa}, \Delta \mathrm{G}_{\mathrm{c}-\mathrm{a}} \sim 5 \mathrm{~kJ} / \mathrm{mol}$ at room temperature). 


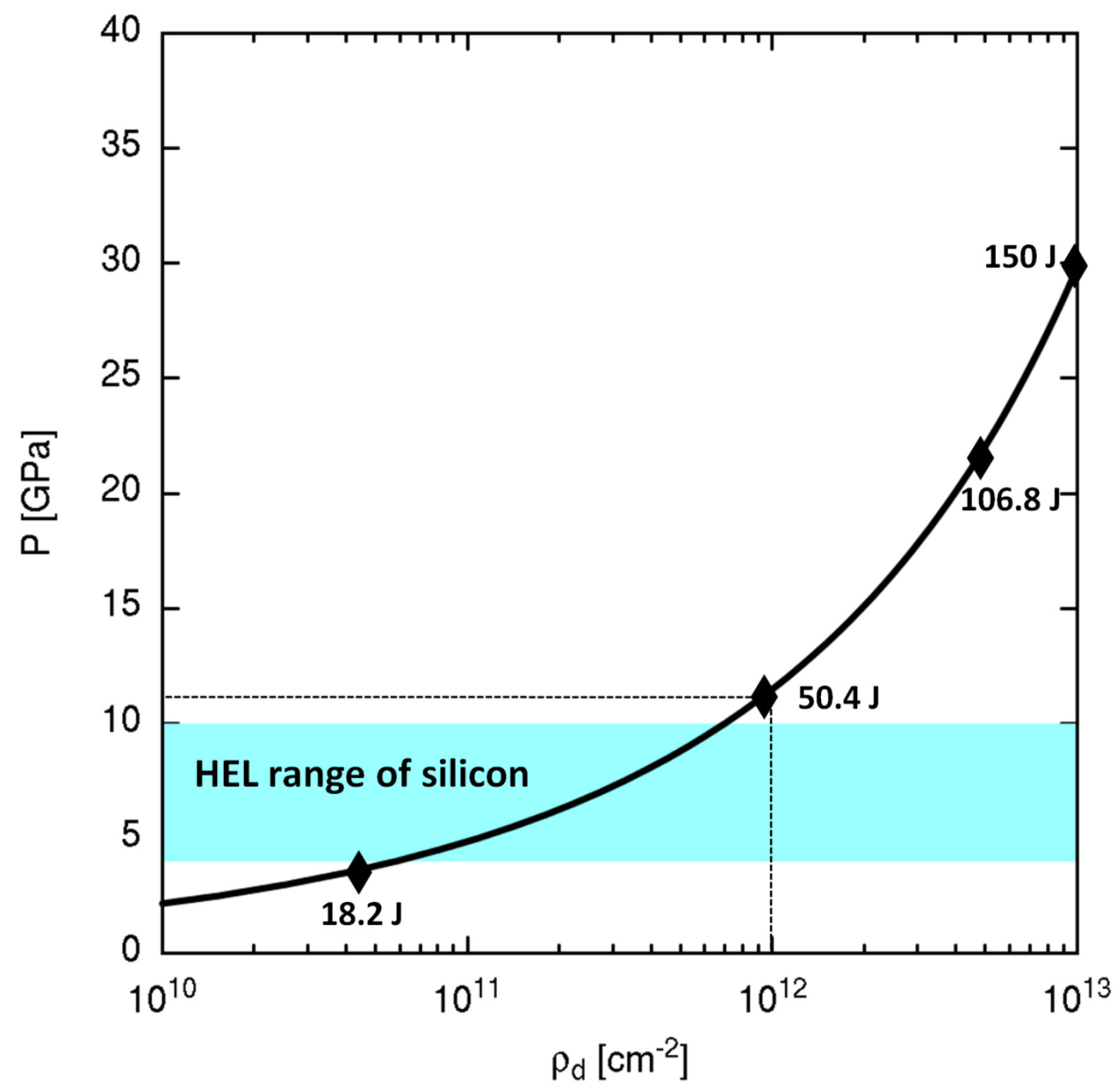

Fig. 14. Calculated dislocation density $\left(\rho_{d}\right)$ as a funcion of pressure $P$ assuming homogeneous nucleation of loops behind front [56]. Hugoniot relationship of the modified Tersoff potential used to obtain pressure vs. volume. Experimental laser experiments marked on plot by their energy levels. The shaded cyan area represents the range of the predicted Hugoniot elastic limit (HEL). The dotted lines denote the shock pressure threshold (and corresponding dislocation density at shock front) where amorphization is observed experimentally. 

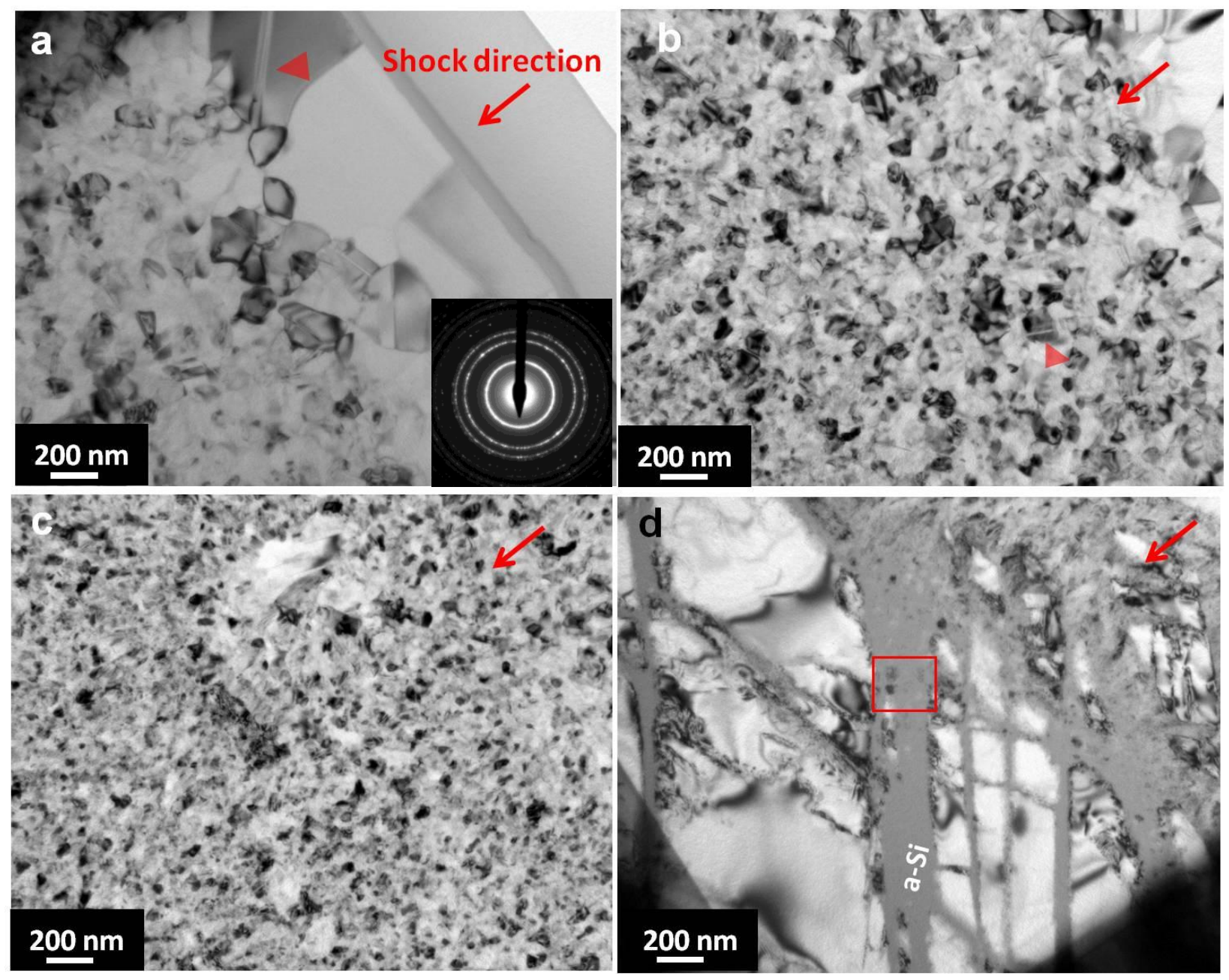

Figure 15. TEM micrographs of the $150 \mathrm{~J}$-shocked silicon crystal showing crystallization of amorphous structure: a, top surface showing grain size of around one micrometer; $b, \sim 3 \mu \mathrm{m}$ below the surface showing a ultrafine grained structure; $\mathrm{c}, \sim 7 \mu \mathrm{m}$ below the surface showing even finer grain size; $\mathrm{d}, \sim 10 \mu \mathrm{m}$ below the surface showing a mixture of amorphous band and undeformed monocrystalline crystal. 


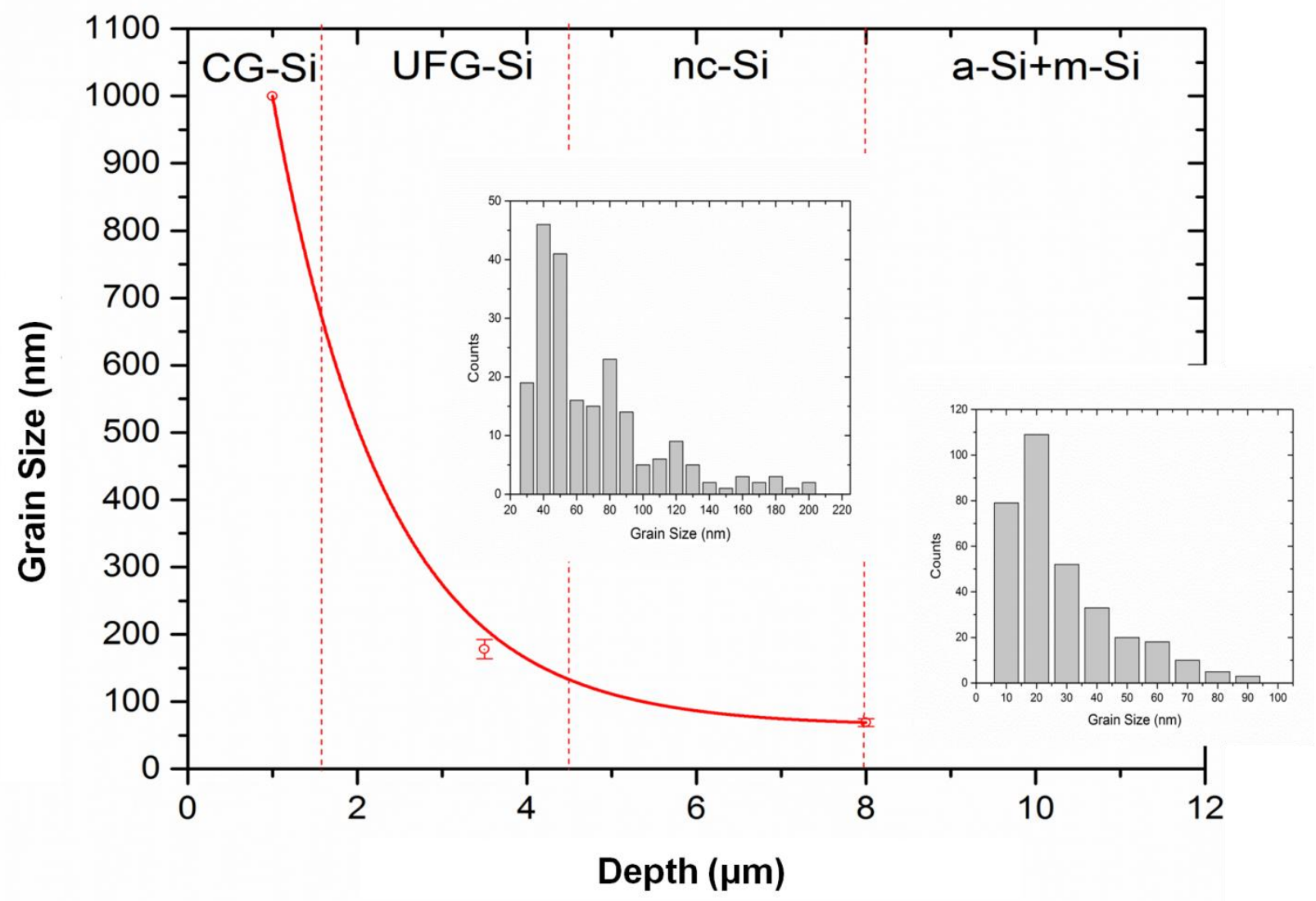

Figure 16. Grain size distribution of the $150 \mathrm{~J}$-shocked sample as function of depth below the shock surface. Four regions, namely coarse grained (CG-Si); ultrafine grained (UFG-Si); nanocrystalline (nc-Si); and mixture of a-Si and monocrystalline (m-Si), can be classified depend on the grain size. 


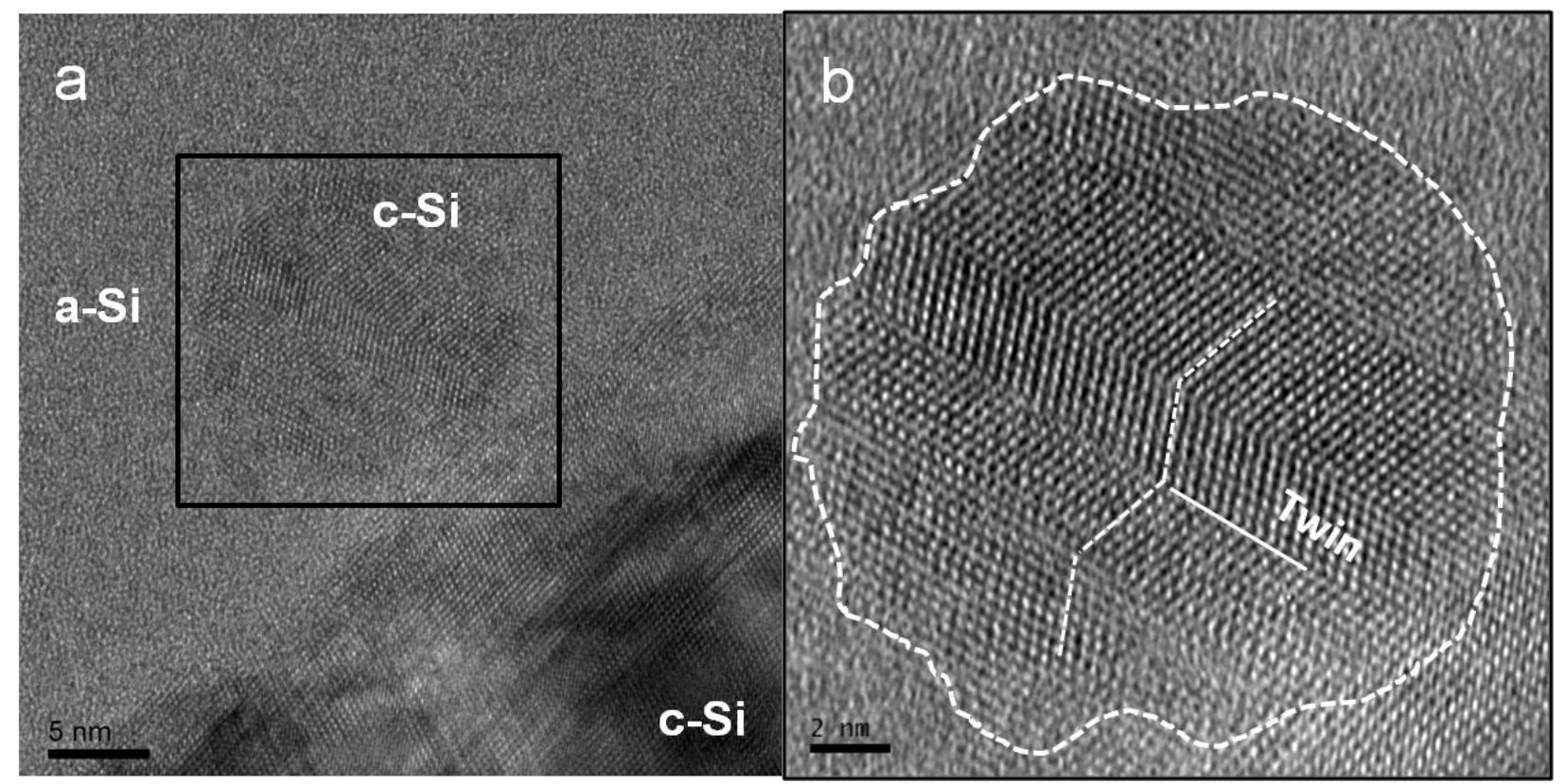

Figure 17. (a) HRTEM micrograph of a nucleus crystallized from amorphous silicon; (b) magnified view of the black boxed region in (a), showing the crystalline embryo (delineated by white dashed line) with clear twined structure. 


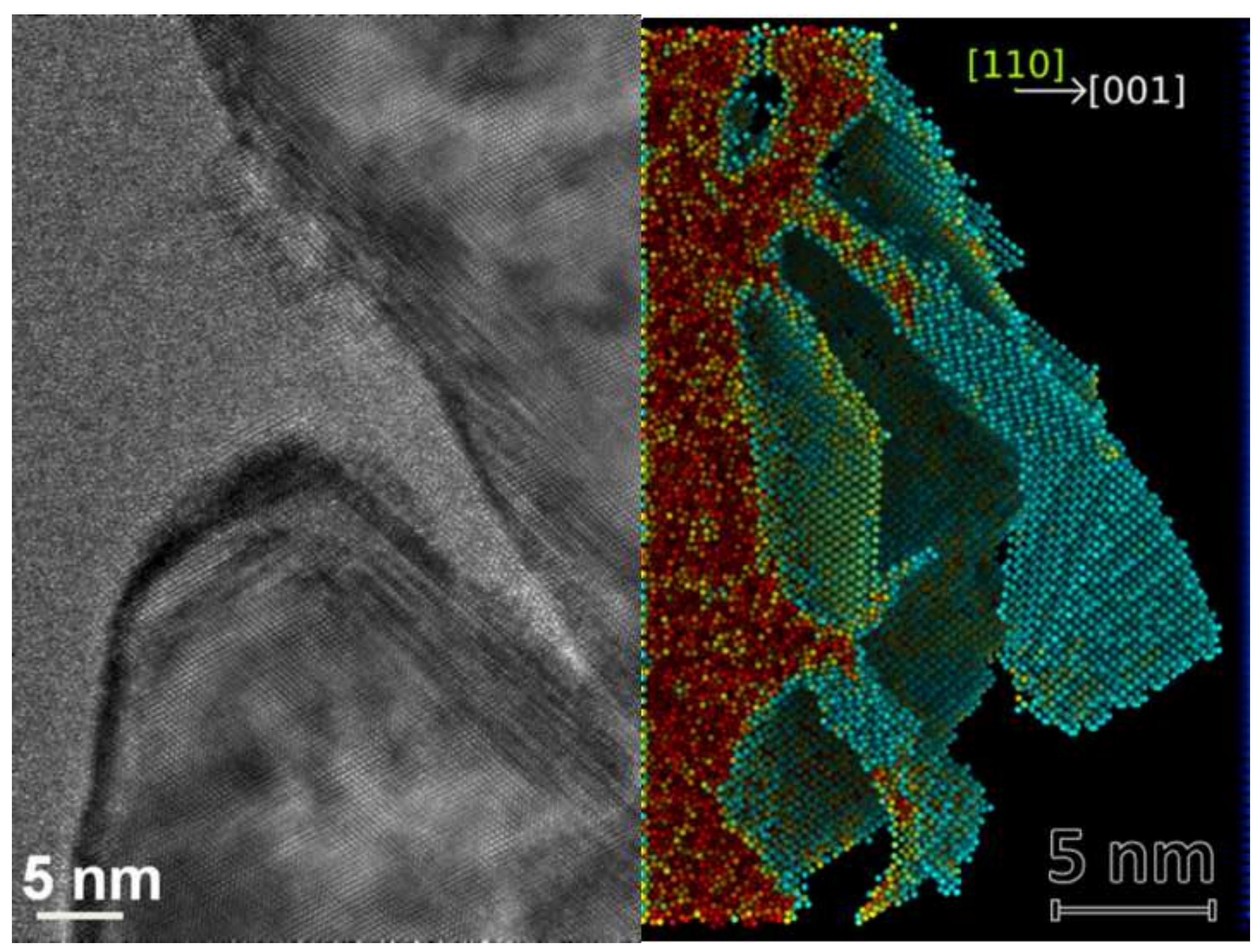

